Research article

urn:1sid:zoobank.org:pub:3FD5D922-B91F-42BB-9D7B-7DFB98841732

\title{
Revision of the genus Intybia (Coleoptera, Malachiidae) from Japan
}

\author{
Hiroshi IKEDA $^{1, *} \&$ Hiroyuki YOSHITOMI ${ }^{2}$ \\ 1,2 Entomological Laboratory, Faculty of Agriculture, Ehime University, \\ Tarumi 3-5-7, Matsuyama, 790-8566 Japan. \\ "Corresponding author: hiroshi0828@gmail.com \\ 2Email: hymushi@agr.ehime-u.ac.jp \\ ${ }^{1}$ urn:Isid:zoobank.org:author:7100DA17-9D15-451B-AEE9-089A1FBF9025 \\ ${ }^{2}$ urn:1sid:zoobank.org:author:744FA6FC-2D84-41B7-8A6C-C1611CC68EE1
}

\begin{abstract}
Japanese species of the genus Intybia are revised taxonomically, with the examination of the endophallic structure. Eight species, including one new species Intybia donan sp. nov. from Yonagunijima, are recognized. All species are described or redescribed with a key and figures. The endophallic structure contains one primary sclerite (gonoporal piece), three secondary sclerites (ligula, semigonoporal piece, and spinous plate) in some species, and a membranous basal area densely covered with many spines (spinous area). Based on the structures of the endophallus, the Japanese members of the genus are divided into two species groups (the histrio and pelegrini groups). The pelegrini species group is furthermore subdivided into three subgroups (subgroups 1-3). New distributional records are as follows: I. histrio from Hachijô-jima and Tanega-shima; I. niponica from Sakhalin and I. takaraensis from Tokuno-shima and Amami-Ôshima.
\end{abstract}

Keywords. Taxonomy, endophallus, new species, species group, distribution.

Ikeda H. \& Yoshitomi H. 2017. Revision of the genus Intybia (Coleoptera, Malachiidae) from Japan. European Journal of Taxonomy 331: 1-31. https://doi.org/10.5852/ejt.2017.331

\section{Introduction}

The melyrid genus Intybia Pascoe, 1866 is represented by about 90 species from the African and Asian regions (Wittmer 1997; Plonski 2013; private data base). The genus Intybia was synonymized with Laius Guérin-Méneville, 1830 by Champion (1921). Since then numerous species have been described under the genus Laius. Evers (1994) re-diagnosed the genus Laius and recognized the genus Intybia as an independent genus again. This act was immediately accepted and a large number of species has been transferred from the genus Laius to the genus Intybia (Wittmer 1995, 1997, 1999; Yoshitomi \& Lee 2010; Plonski 2013, 2014a, 2014b; Plonski \& Geiser 2014).

Up to now, seven species of this genus are known from Japan, but they have not been well understood. In this present paper, we review the Japanese Intybia with observation of the endophallic structure. 


\section{Material and methods}

General observations and dissections were made under a Leica MZ95 stereo microscope. Microstructures of the dissected parts were studied in pure glycerine under an Olympus BH-2 compound microscope. After observation, the dissected parts were mounted on the same card with the specimen. Photographs were taken under a Leica MZ95 using a microscopy camera system (Nikon DS-Fil-L2), and combined using the automontage software Combine ZM (Alan Hadley, UK). Some structures were observed with a SEM (Hitachi S-225), after coating with gold, and a digital microscope HiROX KH-1300. Images were captured with the 2D measurement software SHX-13M ver. 2.9.0.

The dry specimens were prepared by soaking the whole body in hot water for about $5 \mathrm{~min}$; this process was omitted for the fresh specimens. The genitalia were removed with twizzers under a Leica MZ95 stereo microscope.

The method of observation of the endophallic structures was the same as in Yoshitomi (2014).

Distribution of each species follows Mayor (2007), Yoshitomi \& Hayashi (2011) and Korean National Arboretum (2014). New distributional records are indicated as "[NR]" after the locality name.

The specimens used in this paper are preserved in the following institutions and private collections:

EUMJ = Ehime University Museum, Matsuyama, Japan

SEHU $=$ Hokkaido University Museum, Hokkaido, Japan

KUM $=$ Kyushu University Museum, Fukuoka, Japan

HSJ = private collection of H. Suenaga, Japan

NTJ = private collection of N. Tsuji, Japan

YOJ = private collection of Y. Ogata, Japan

\section{Characteristics and terminology of endophallic structures}

The terminology of the endophallic structures mainly follows Yoshitomi (2014), who studied the related genus Laius, although the constructions of the endophallus in the genus Intybia are somewhat different from those of Laius. In the present paper, we use the following technical terms for the endophallic structures (see also Fig. 7).

Spinous area (after Yoshitomi 2014; Fig. 7B): membranous basal area, densely covered with many short spines, about $0.5 \times$ length of AL.

Spinous plate(s) (named in this paper; Fig. 8B-C): 1 or 2 small sclerotized plate(s) situated in the basal part of the gonoporal piece, densely covered with many spines.

Gonoporal piece (after Yoshitomi 2014; Fig. 7B-C): longest sclerite, about $0.5 \times$ length of AL, straight to rather curved, with pointed or rounded apex, covered with minute spines in the middle portion of some species, projecting apically and straight from the apical portion.

Semi-gonoporal piece (named in this paper; Fig. 7B, D): long sclerite, shorter than the gonoporal piece, straight to rather curved, with pointed apex, projecting apically and straight from the apical portion.

Ligula (after Yoshitomi 2014; Fig. 7B, E): shortest sclerite, straight to rather curved, with pointed apex, projecting laterally from the basal portion of the gonoporal piece.

\section{Abbreviations}

Morphological abbreviations used for measurements are as follows.

$\mathrm{AL}=$ aedeagus length

$\mathrm{EL}=$ length of elytra in suture 


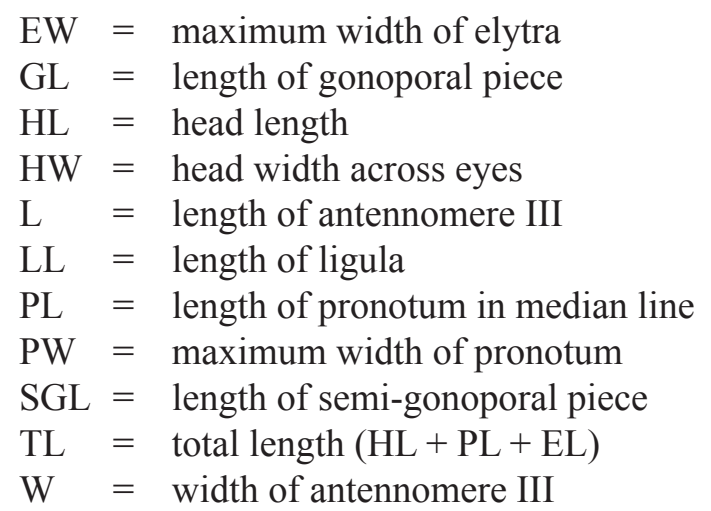

The average is given in parentheses after the range.

\section{Results}

Class Hexapoda Blainville, 1816

Order Coleoptera Linnaeus, 1758

Superfamily Cleroidea Latreille, 1802

Family Malachiidae Fleming, 1821

Tribe Apalochrini Muslant \& Rey, 1867

Genus Intybia Pascoe, 1866

Intybia Pascoe, 1866: 448 [type species: Intybia guttata Pascoe, 1866].

\section{Notes}

The genus Intybia belongs to the tribe Apalochrini of the subfamily Malachiinae together with the related genera Laius and Dicranolaius (Champion, 1921). The genus Intybia is characterized as follows: elytra with yellowish to reddish fascia or maculation in most species (vs fully black with bluish luster in Laius); antennomere II short; antennomere III in male modified; simple protarsus and protibia in male (vs two tarsal combs in protarsi V in Dicranolaius); front legs slender (vs male protibia of Laius thickened basally and inside of basal area distinctly hollow); aedeagal apex round in Japanese species (vs concave in most Laius species); endophallic structure composed of 1-3 sclerites (gonoporal piece, semi-gonoporal piece, ligula, and 0-2 spinous plate(s)) (Evers 1994; Asano \& Kawashima 2010; Asano 2015).

\section{Gender}

Feminine.

\section{Key to the Japanese species of the genus Intybia}

1. Elytra with fascia, but sometimes fascia divided into two spots by suture; distributed in Hokkaido to Ryukyu I. donan sp. nov.

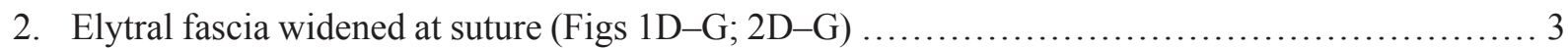

- Elytral fascia narrowed at suture, or divided into two spots by suture (Figs 1A, B, H; 2A-B, H)..6

3. Fore legs fully yellow; head wide, about $0.5-0.9 \mathrm{in} \mathrm{HL/HW;} \mathrm{eyes} \mathrm{prominent;} \mathrm{endophallus} \mathrm{with} \mathrm{spinous}$

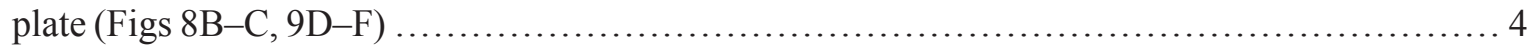


- Fore legs fully black; head narrow, about $0.9-1.2$ in HL/HW; eyes not prominent; spinous plate absent (Figs 8D,9G) I. kishiii(Nakane, 1955)

4. Body oval; femur black; endophallus with one spinous plate (Figs 8C, 9E-F) ........... 5

- Body elongate; femur yellow; endophallus with two spinous plates (Figs 8B, 9D).....

I. pelegrini pelegrini (Pic, 1910)

5. Elytral fascia clearly widened at suture; body black; male antennomere III broad bean-like shaped; female apterous....

I. niponica (Lewis, 1895)

- Elytral fascia slightly widened at suture; body black with bluish luster; male antennomere III heartlike shaped; female hind wing present

I. takaraensis (Nakane, 1955)

6. Fore legs fully yellow; elytra with a yellow or divided into two spots by suture

- Fore legs fully black; elytra with a fascia

I. tsushimensis (Satô \& Ohbayashi, 1968)

7. Elytral fascia varies in development (Fig. 3), sometimes interrupted by suture; antennae yellow; male antennomere III fusiform, with an oval funnel-shaped on the outer edge, with a twisted projection near base; male frons yellow in apical half .................... I. histrio (Kiesenwetter, 1874)

- Elytral fascia interrupted by suture; antennomeres V-XI infuscate; male antennomere III ear-like shaped; male frons fully black I. kawasakii (Nakane, 1956)

Intybia histrio (Kiesenwetter, 1874)

[Japanese name: Hiro-obi-jôkai-modoki]

Figs 1A, 2A, 3, 4A, 5A, 6A, 7, 8A, 9A, 10A, 11A, 12A

Lajus [sic] histrio Kiesenwetter, 1874: 283 [type locality: Kiushiu].

Lajus [sic] flavicornis Kiesenwetter, 1874: 283 [nec Fabricius, 1801]. Synonymized by Lewis (1893).

Laius kiesenwetteri Lewis, 1893: 151 (replacement name for Lajus [sic] flavicornis Kiesenwetter, 1874).

Laius histrio - Champion 1921: 151. — Nakane 1963: 186, pl. 93, fig. 13. — Nakane 1957: 13, fig. 1. — Nakane 1983: 164.

Intybia histrio -Wittmer 1997: 191. — Mayor 2007: 417 [catalogued]. — Tshernyshev 2009: 35, figs 8-15. - Yoshitomi \& Hayashi 2011: 19. - Tshernyshev 2012: 575, table 1.

Laius historio - Satô 1985: 165, pl. 26, fig. 9. — Satô 1989: 366 [misspelling].

Laius kiesenwetteri - Lewis 1895: 116.

\section{Diagnosis}

Antennae entirely yellow; head yellow in apical half; elytral fascia wide, but variable (Fig. 3); antennomere I long, abruptly widened from near the apical portion; antennomere III fusiform, with an oval funnel-shape on outer edge, with a twisted projection near base; gonoporal piece curved, widened from near apical portion, with straight basal projection, bearing minute spines on edge; semi-gonoporal piece straight, slender, widened near central part; ligula long, straight; spinous plate absent.

\section{Material examined}

JAPAN, Honshu: Tochigi Pref.: 1 ô, Ôhira-san, Sonobe, 11 Aug. 1980, H. Kurokawa leg. (EUMJ); 1 q, Ôhira-san, Sonobe, 23 Aug. 1988, H. Yoshitomi leg. (EUMJ); 1 ô, Katayama Park, Kuzuu, 25 Sep. 1980,

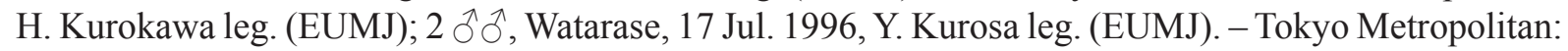
1 9, Toshima, 27 Jul. 1950, S. Hisamatsu leg. (EUMJ); 1 ô, Narimasu, 12 Jul. 1984, collector unknown (EUMJ). - Kanagawa Pref.: 18 $\widehat{\jmath}$, Kanazawa-ku, Yokohama, Jul. 1976, Hasegawa leg. (EUMJ); 1

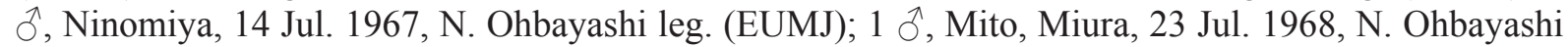




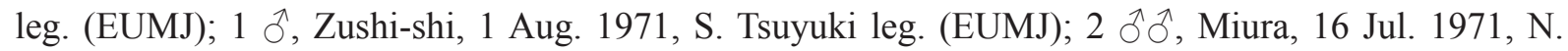
Ohbayashi leg. (EUMJ); 1 đ̃, 1 क, Hiratsuka, 6 Jul. 1978, Y. Notsu leg. (EUMJ). - Shizuoka Pref.: 1 q, Okitsu, Seikenji, Sizuoka-shi, 30 Aug. 2004, Y. Satô leg. (EUMJ). - Aichi Pref.: 1 ô, Toyokawa, S-2, 3 Aug. 1995, (EUMJ); 1 ô, Toyokawa, S-2, 3 Aug. 1995, (EUMJ); 1 ô, Chita, st. 2, Minamihama-chô, Chita-shi, 15 Jul. 1982, B. Tanaka leg. (EUMJ); 1 Ô, Meijo Univ., Nagoya-shi, Apr. 1991, H. Yoshitomi leg. (EUMJ). - Mie Pref.: 1 §, Yokkaichi, Aug. 1979, M. Nagai leg. (EUMJ). - Hyogo Pref.: 1 ठૈ, Karasuhara, Kôbe-shi, 26 Jul. 1953, T. Takahashi leg. (EUMJ); 1 đ̂, 1 ㅇ, Akashi-shi, 24 Jul. 1970, M. Tomokuni leg. (EUMJ); 1 ô, 1 q, Maya-san, 16 Jul. 1948, S. Iwao leg. (EUMJ). - Tottori Pref.: 1 \%, Hoki-chô, Iwatate, Masumizu-Kôgen, 20 Jul. 2013, H. Suenaga leg. (HSJ). - Okayama Pref.: 1 q, Higashiazo, Sôja-shi, 15 Aug. 1995, H. Ono leg. (EUMJ); 1 ô, Kurashiki-shi, Koseto, 6 Sep. 2009, H.

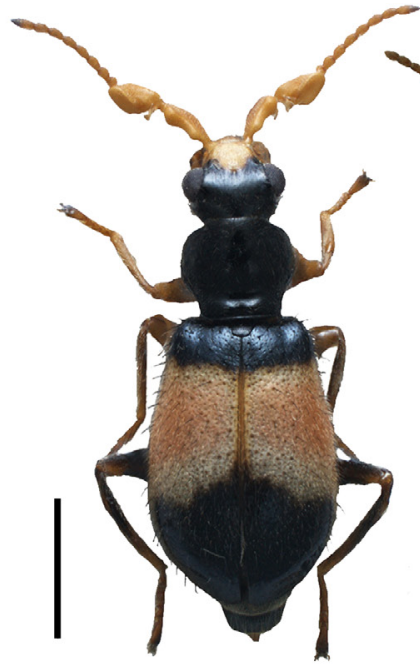

A

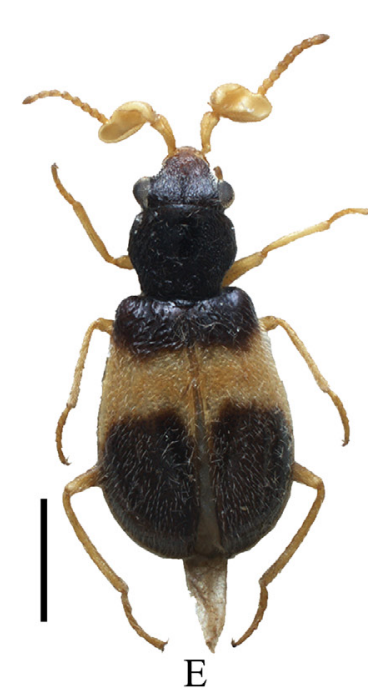

$\mathrm{E}$

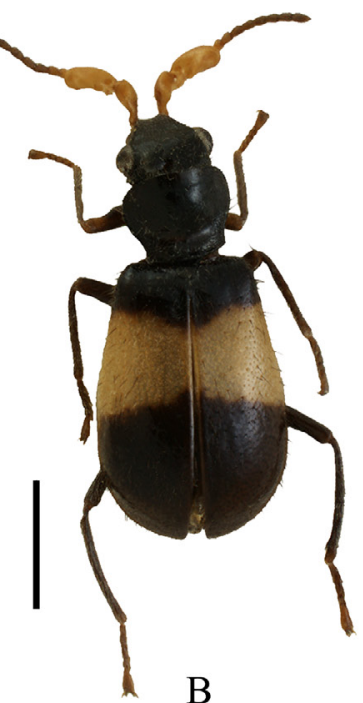

B

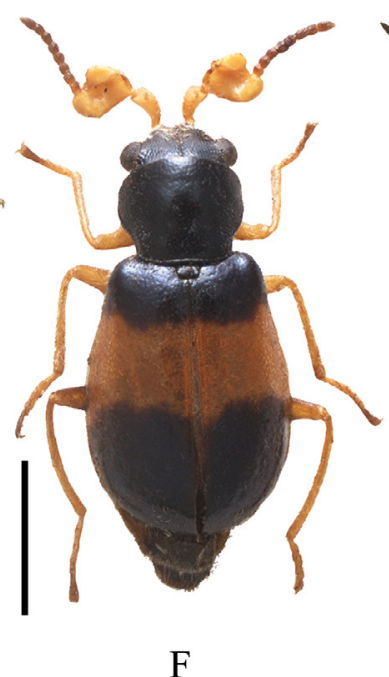

$\mathrm{F}$

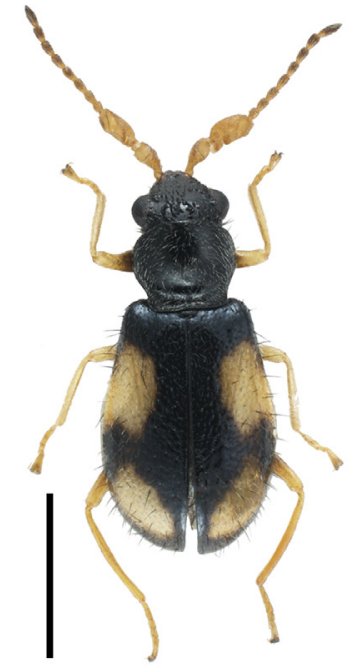

$\mathrm{C}$

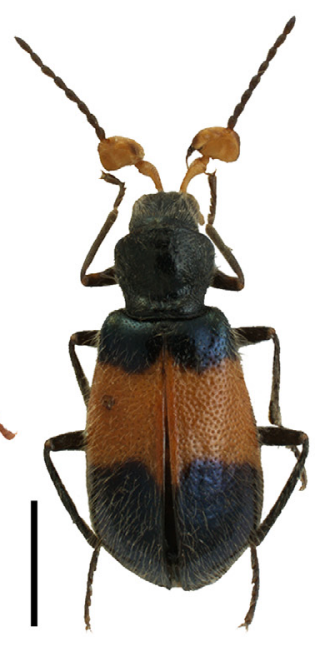

G

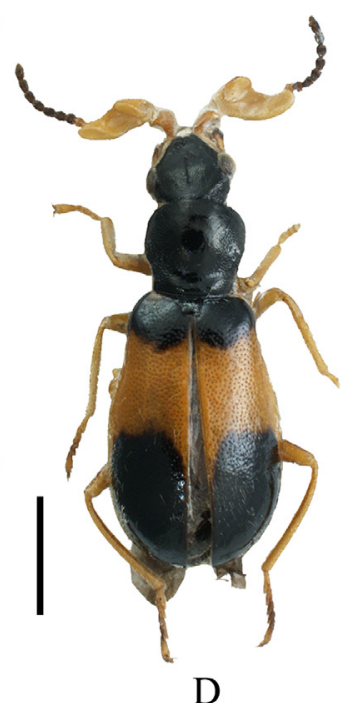

$\mathrm{D}$

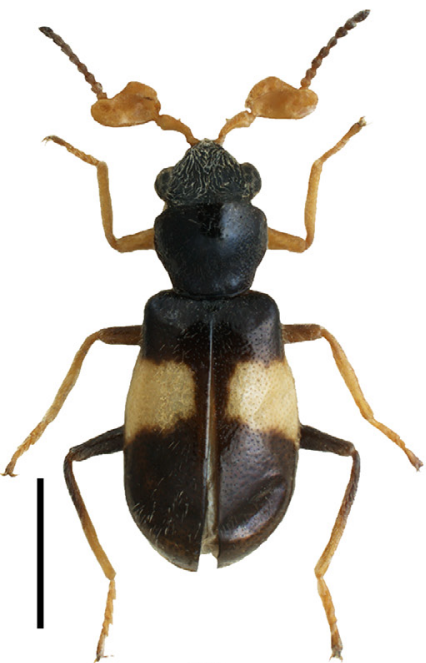

$\mathrm{H}$

Fig. 1. Dorsal habitus Intybia spp., Oे. A. Intybia histrio (Kiesenwetter, 1874). B. I. tsushimensis (Satô \& Ohbayashi, 1968). C. I. donan sp. nov., holotype. D. I. pelegrini pelegrini (Pic, 1910). E. I. niponica (Lewis, 1895). F. I. takaraensis (Nakane, 1955). G. I. kishiii (Nakane, 1955). H. I. kawasakii (Nakane, 1956), holotype. Scales $=1.0 \mathrm{~mm}$. 
Suenaga leg. (HSJ). - Yamaguchi Pref.: 1 ㅇ, Shizukisan, Hagi, 9 Sep. 1970, S. Hisamatsu leg. (EUMJ); 1 +, Shizukisan, Hagi, 14 Sep. 1973, S. Hisamatsu leg. (EUMJ).

JAPAN, Shikoku: Tokushima Pref.: 1 + , Kawata-kitajima, Yamakawa-chô, 23-26 Aug. 1999, T. Doi and K. Aita leg. (EUMJ); 1 + , mouth of Naka-gawa, Nakagawa-chô, 12-14 Oct. 2000, K. Aita leg. (EUMJ); 1 §ె, Kawata-kitajima, Yamakawa-chô, 23-26 Aug. 1999, T. Doi and K. Aita leg. (EUMJ). -

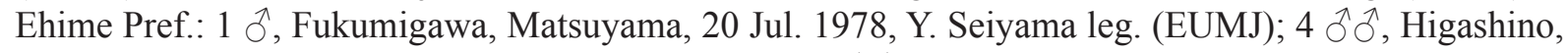
Matsuyama-shi, Jul. 2015, K. Sogoh leg. (EUMJ); 4 đ̊̄, Ishitegawa, Matsuyama-shi, 30 Jun. 2015, H. Ikeda leg. (EUMJ); 1 đ̃, Jikiba, Matsuyama-shi, 22 Jun. 2004, Y. Satô leg. (EUMJ); 1 + , Gion-machi, Matsuyama, 20 Jul. 2003, M. Sakai leg. (EUMJ); 1 ㅇ, Shimoidai, Matsuyama, 9 Jul. 1998, S. Hisamatsu leg. (EUMJ); 1 + , Shôhachiman-Shrine, Fukuzumi-chô, Matsuyama, 22 Jul. 1992, S. Hisamatsu leg. (EUMJ); 1 ㅇ, Sugitate, Matsuyama-shi, 12 Jul. 1975, A. Oda leg. (EUMJ); 1 §, Sugitate, Matsuyamashi, 30 Jun. 1997, S. Hisamatsu leg. (EUMJ); 1 đ̂, Sugitate, Matsuyama-shi, 7 Jun. 1979, T. Inakura

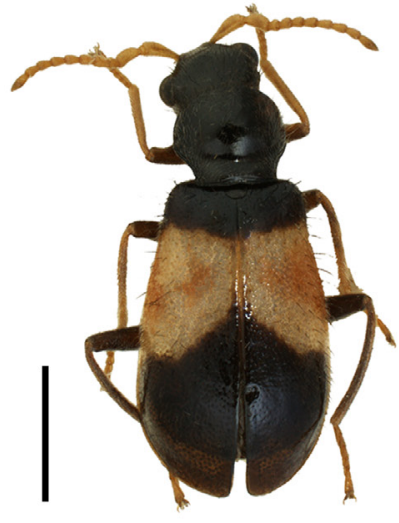

A

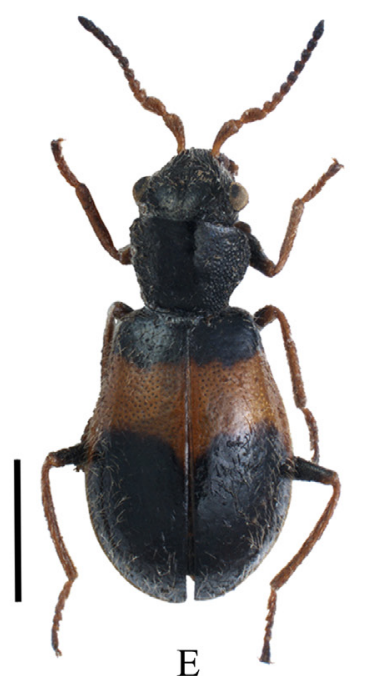

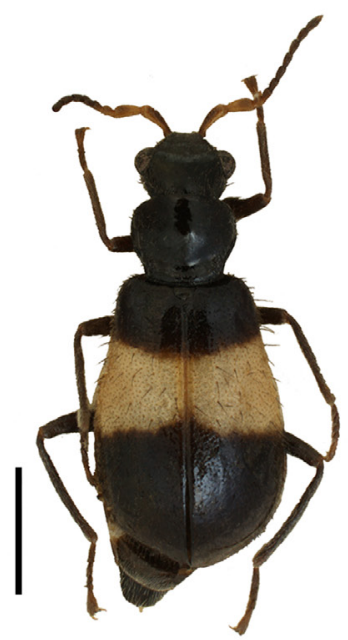

B

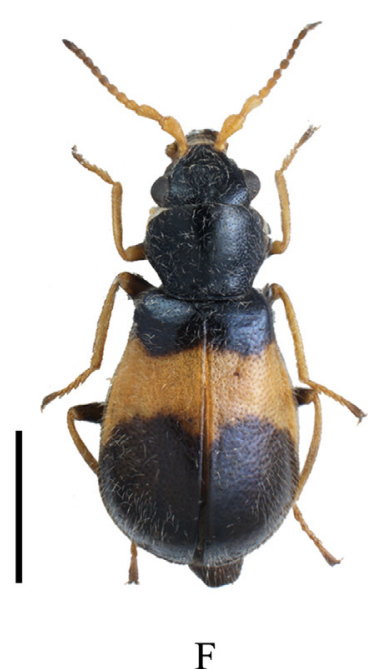

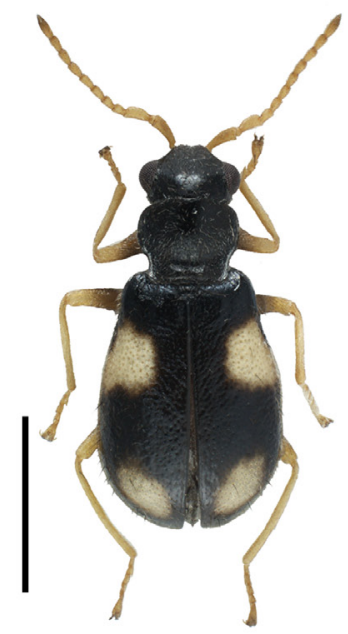

$\mathrm{C}$

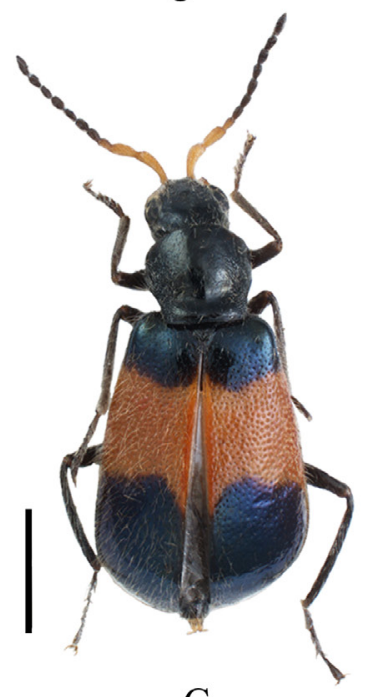

G
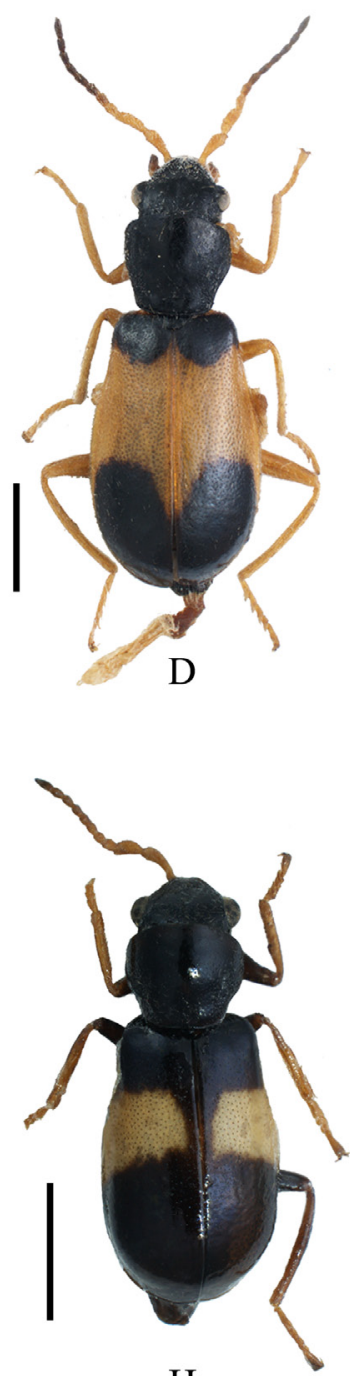

$\mathrm{H}$

Fig. 2. Dosal habitus of Intybia spp., . . A. Intybia histrio (Kiesenwetter, 1874). B. I. tsushimensis (Satô \& Ohbayashi, 1968). C. I. donan sp. nov., paratype. D. I. pelegrini pelegrini (Pic, 1910). E. I. niponica (Lewis, 1895). F. I. takaraensis (Nakane, 1955). G. I. kishiii (Nakane, 1955). H. I. kawasakii (Nakane, 1956), paratype. Scale bars $=1.0 \mathrm{~mm}$. 
leg. (EUMJ); $1 \hat{\partial}, 1$ क, Sugitate, Matsuyama-shi, 15 Jul. 2004, T. Kitano leg. (EUMJ); $2 \hat{\partial} \widehat{\partial}$, Tamatanimachi, Matsuyama-shi, 12 Jul. 2005, T. Kitano leg. (EUMJ); 1 §, Tarumi, Matsuyama-shi, 7 Jul. 2004

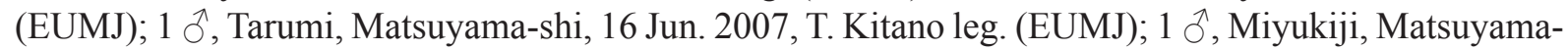

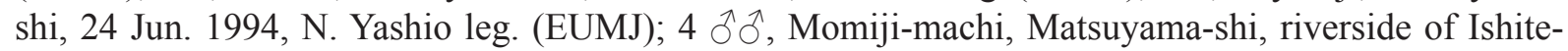
gawa, 24 Jun. 2015, Y. Hisasue leg. (EUMJ); $76 \partial^{\lambda} \sigma^{\lambda}$, same data, 25 Jun. 2015, H. Ikeda leg. (EUMJ);

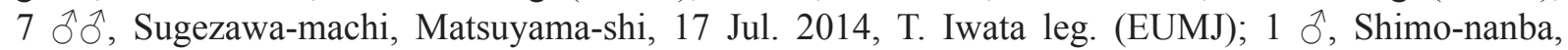
Matsuyama-shi, 3 Sep. 2009, L. Takachi leg. (EUMJ); 4 ồ ô, Jyôkoji-machi, Matsuyama-shi, 3350'58" N, 132 47'53" E, alt. 100-120 m, 26 Jun. 2007, T. Kurihara leg. (EUMJ); 1 q, Imabari-shi, Ônishi-chô, Ôihama, 14 Nov. 2006, Y. Satô leg. (EUMJ); 1 ㅇ, Iyo-shi, 8 Jul. 1975, M. Sakai leg. (EUMJ); 1 ㅇ, Iyo-shi, Shimo-kawara, 16 Sep. 2005, T. Kurihara leg. (EUMJ); 1 đ, Wadamaru, Sunouchi, Toon-shi, 25-30 Jun. 2007, J. Ogawa leg. (EUMJ); 1 Oૈ, Sankason-izumi, Shigenobu-chô, 17 Jun. 1998, K. Aita

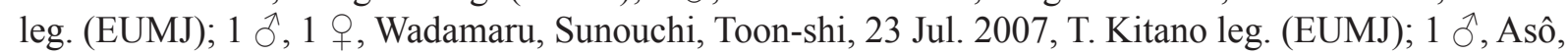
Tobe-chô, side of Nishinotani Ponds, 3 Jul. 1998, S. Hisamatsu leg. (EUMJ); 1 Ô., Tobe-chô, Iyo-gun, 24 Jun. 1980, K. Sasagawa leg. (EUMJ); 1 đે, Nakamura, Ôzu shi, 20 Jun. 2001, M. Sakai leg. (EUMJ);

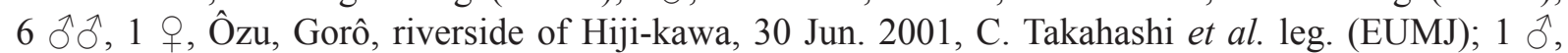

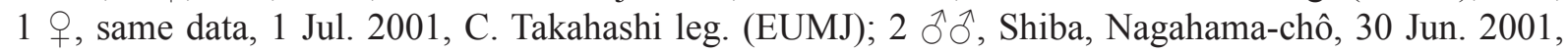
H. Ishikawa et al. leg. (EUMJ); $1 \hat{\jmath}$, same data, 31 Jun. 2001, Y. Kikuhara leg. (EUMJ); $1 \hat{\jmath}$, same

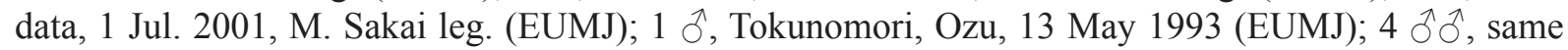
data, 19 Jul. 1993 (EUMJ); 1 +, same data, 24 Aug. 1993 (EUMJ); 1 q, Kumakôgen-chô, Saragamine, 1 Jun. 2008, Y. Satô leg. (EUMJ); 1 đ, Koya-yama, Odamiyama, 23 Jul. 1996, H. Ono leg. (EUMJ); 1 ô, Namakusa-dani, Odamiyama, 20 Jul. 1994, E. Yamamoto leg. (EUMJ); 2 q + , Oda-chô, 24 Jul. 1994, E. Yamamoto leg. (EUMJ); 1 ô, Yoshinogawa, Oda-chô, 21 Jul. 1993, K. Aita leg. (EUMJ); 7 우, Nomura Dam, Nomura, 23-24 Jul. 1994, Ohbayashi, Okada, Fujioka leg. (EUMJ); 6 우, same data, 23 Jul. 1994, M. Sakai leg. (EUMJ); 1 क, same data, 24 Jul. 1994, M. Sakai leg. (EUMJ); 1 đૈ, 2 우, Tawarazu, Akehama, Seiyo, 12 Jul. 2008, J. Ogawa leg. (EUMJ); 1 ô, Yamada, Seiyo-shi, Uwachô, 19 Jun. 2004, T. Kurihara leg. (EUMJ); 1 đે, Ryutakuji-ike, Shirokawa-chô, Uonashi, Seiyo-shi, 10 Jul. 2006, J. Ogawa leg. (EUMJ); 1 ô, Hokezu, Yoshida, Uwajima-shi, 12 Jul. 2008, L. Takechi leg. (EUMJ); 1 q, Iyo-Uwa, 5 Aug. 1956 (EUMJ); 1 \&, Shiroyama, Uwajima, 19 Jul. 1955, S. Ueda and

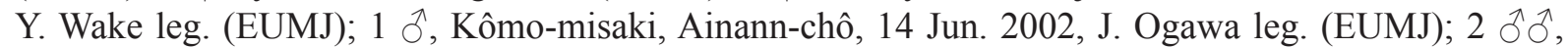
2 오, same data, 30 Jun. 2004, J. Ogawa leg. (EUMJ); 1 ㅇ, same data, 18 Jul. 2005, J. Ogawa leg.

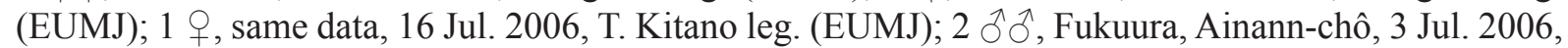
J. Ogawa leg. (EUMJ); 1 + , same data, 30 Jun. 2004, J. Ogawa leg. (EUMJ); $2 \hat{\jmath} \hat{\jmath}, 3$ 웅, Hisayoshi, Jyôhen-chô, 30 Jun. 2004, J. Ogawa leg. (EUMJ); 3 đ̂̉, Ôhama, Jyôhen-chô, 4 Jul. 2000, J. Ogawa leg. (EUMJ); 1 §, Sunokawa, 20-21 Jul. 1967, M. Miyatake, M. Iga and H. Ohnishi leg. (EUMJ);
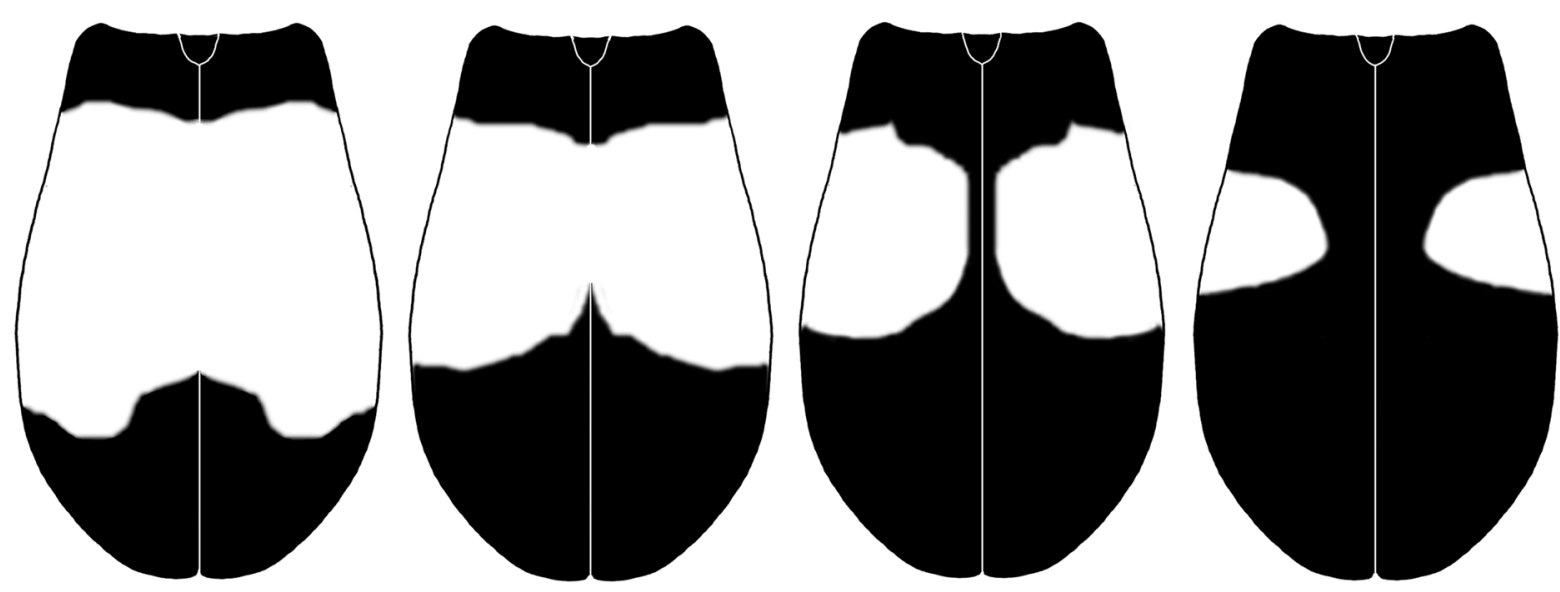

Fig. 3. Elytral pattern variation of Intybia histrio (Kiesenwetter, 1874). 


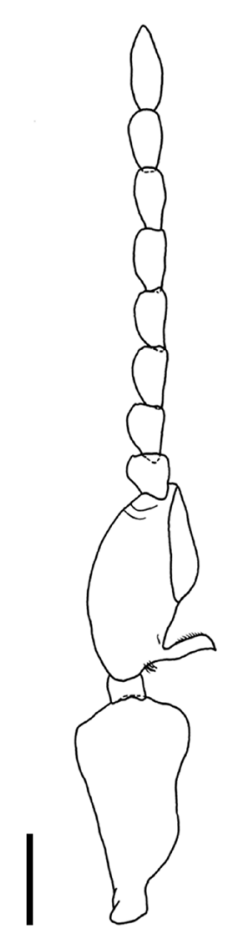

A

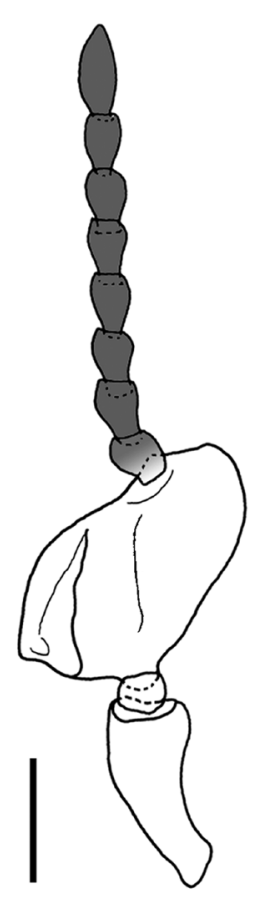

$\mathrm{E}$

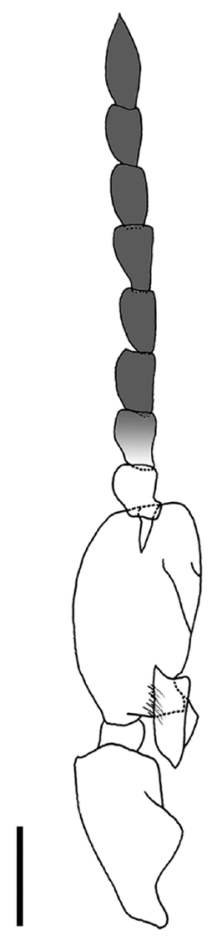

B

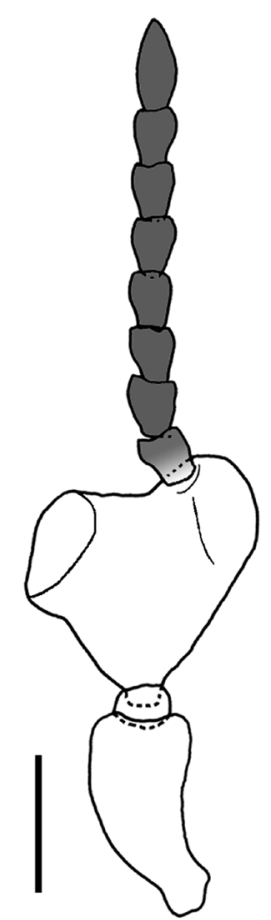

$\mathrm{F}$

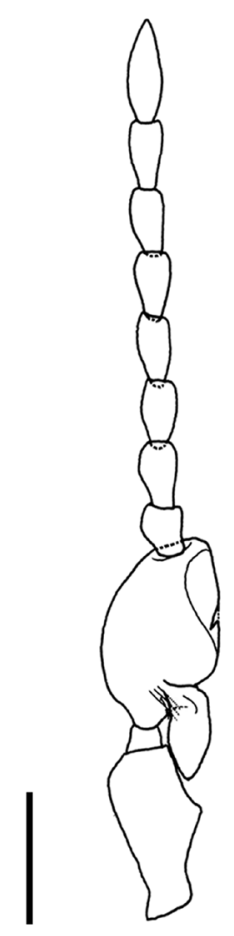

$\mathrm{C}$

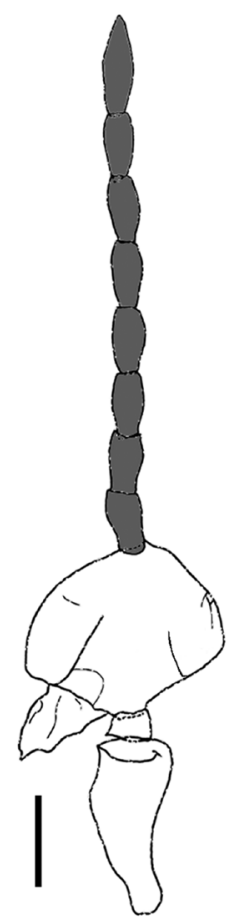

G

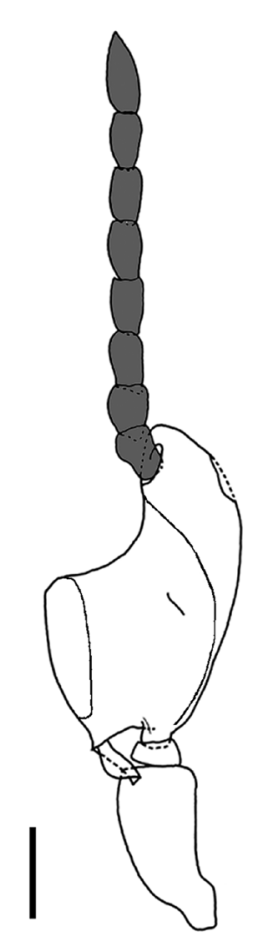

$\mathrm{D}$

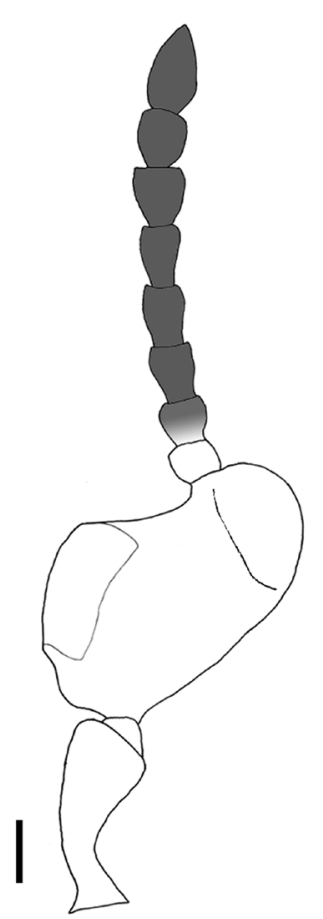

$\mathrm{H}$

Fig. 4. Right antennae of Intybia spp., Ô. A. Intybia histrio (Kiesenwetter, 1874). B. I. tsushimensis (Satô \& Ohbayashi, 1968). C. I. donan sp. nov. D. I. pelegrini pelegrini (Pic, 1910). E. I. niponica (Lewis, 1895). F. I. takaraensis (Nakane, 1955). G. I. kishiii (Nakane, 1955). H. I. kawasakii (Nakane, 1956). Scale bars $=0.2 \mathrm{~mm}$. 


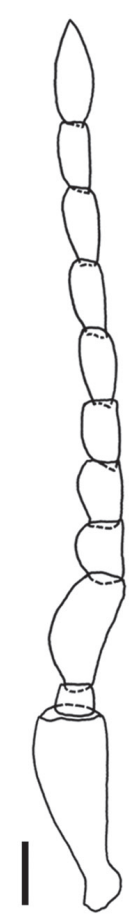

A

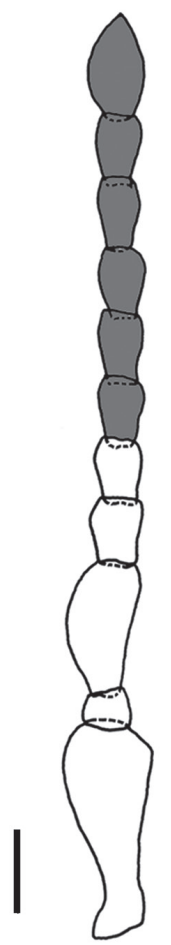

$\mathrm{E}$

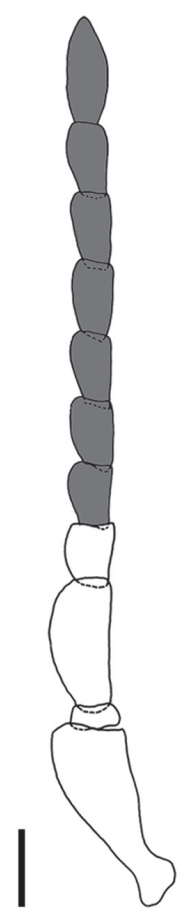

B

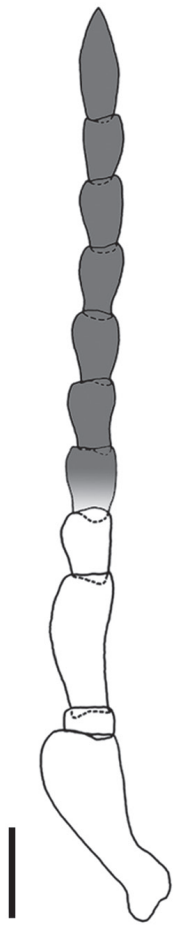

$\mathrm{F}$

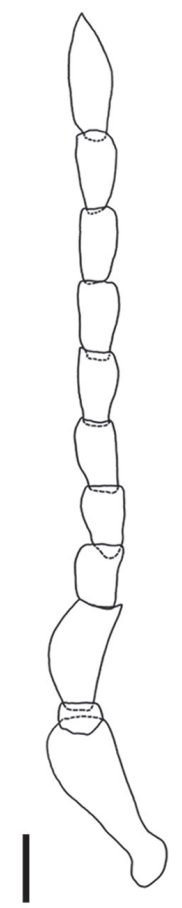

$\mathrm{C}$

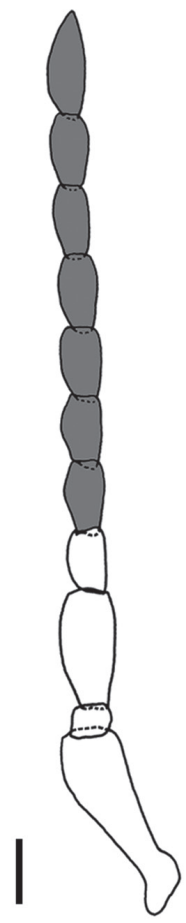

G

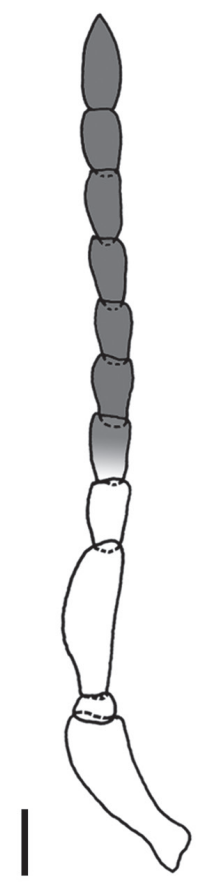

$\mathrm{D}$

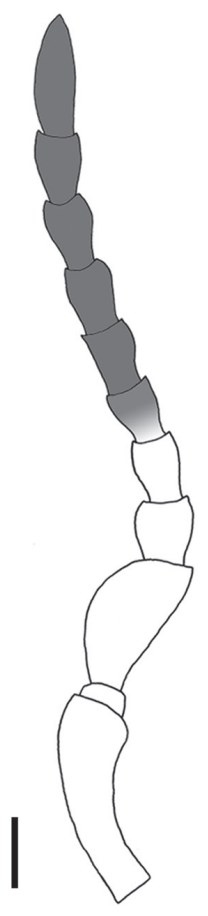

$\mathrm{H}$

Fig. 5. Right antennae of Intybia spp., ․ A. Intybia histrio (Kiesenwetter, 1874). B. I. tsushimensis (Satô \& Ohbayashi, 1968). C. I. donan sp. nov. D. I. pelegrini pelegrini (Pic, 1910). E. I. niponica (Lewis, 1895). F. I. takaraensis (Nakane, 1955). G. I. kishiii (Nakane, 1955). H. I. kawasakii (Nakane, 1956). Scale bars $=0.2 \mathrm{~mm}$. 

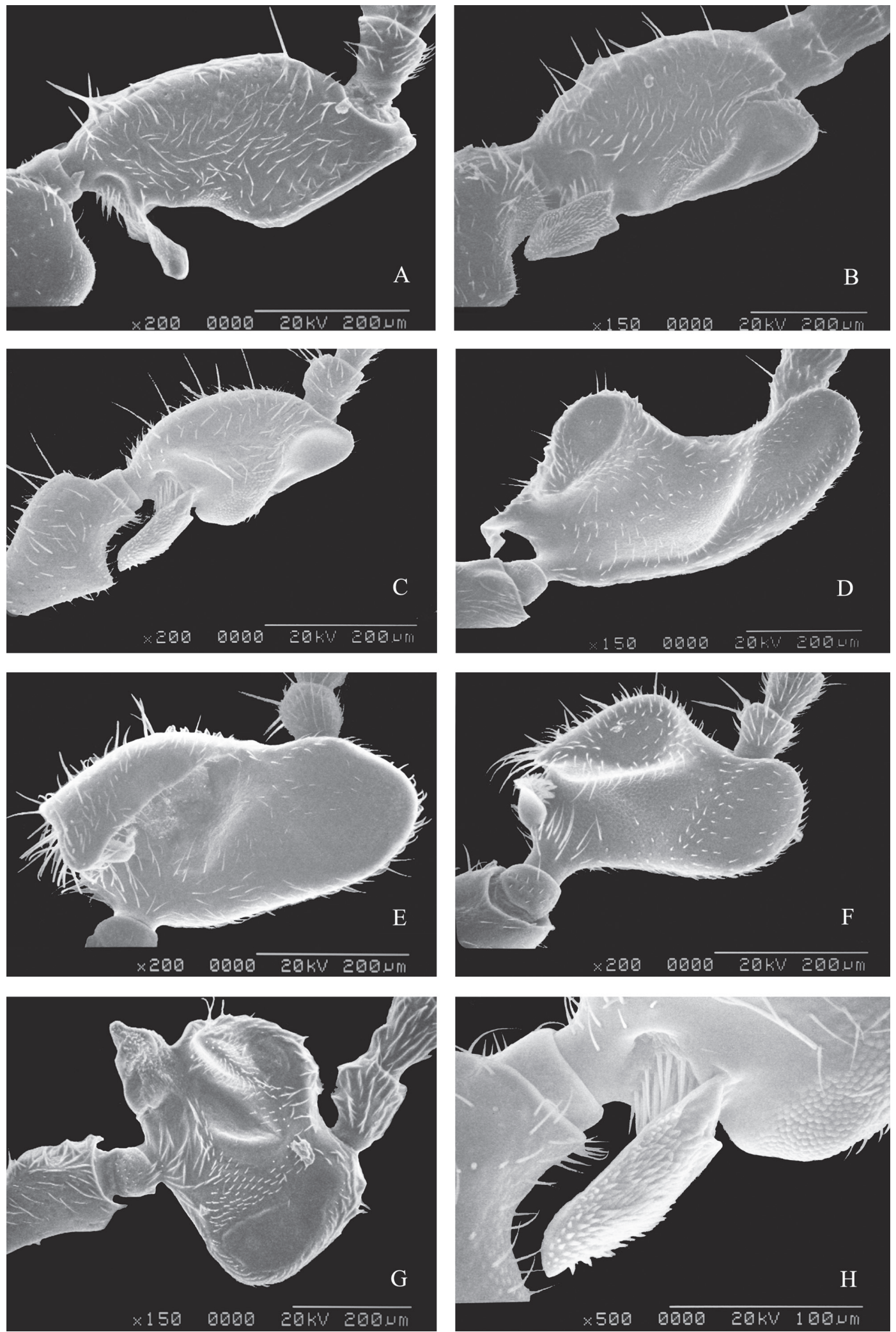

Fig. 6. SEM photographs of right male antennomere III of Intybia spp. A. Intybia histrio (Kiesenwetter, 1874). B. I. tsushimensis (Satô \& Ohbayashi, 1968). C, H. I. donan sp. nov., showing projection (H). D. I. pelegrini pelegrini (Pic, 1910). E. I. niponica (Lewis, 1895). F. I. takaraensis (Nakane, 1955). G. I. kishiii (Nakane, 1955). 
1 q, same data, 16 Jul. 1967, S. Hisamatsu leg. (EUMJ); 7 đô, 7 q $q$, Akahone-jima, Kamishima-chô,

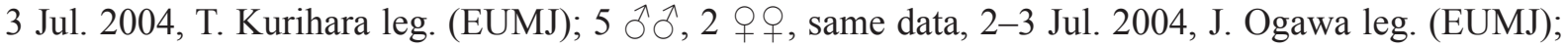
5 ふึત, 2 웅, same data, 2-3 Jul. 2004, N. Ohbayashi leg. (EUMJ); 1 ㅇ, same data, 27-28 Aug. 2004, J. Yamasako leg. (EUMJ); 2 우, same data, 13 Jul. 2006, J. Ogawa leg. (EUMJ); 4 우, same data,

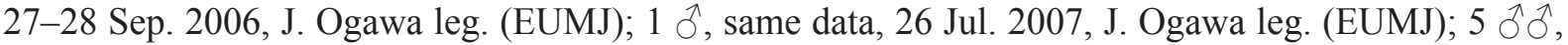
6 우 , Hakatajima, 1 Aug. 2015, H. Yoshitomi leg. (EUMJ); 1 , Aijima, Hojo-shi, 16 Aug. 1976, M. Miyatake leg. (EUMJ); 1 +, Nakajima, 12 Apr. 1958, F. Takechi leg.; 1 O, 2 웅, Is. Nuwa, 29 Aug. 1957, F. Takechi leg.; 1 q, Kashima, Hojo-shi, 27 Jul. 2002, Y. Kikuhara leg. (EUMJ); 1 ô, 1 \&, Ôdô near Kashiwajima, 13 Jul. 1987, Y. Utsunomiya leg.; 1 §̂, Oshima, Yosokuni, Miyakubo-chô, Imabarishi, 13 Jul. 2006, J. Ogawa leg. (EUMJ). - Kochi Pref.: 3 우, Ashizuri-misaki, Tosashimizu-shi, 12 Jul. 1987, M. Sakai leg. (EUMJ); 1 Ô, same data, 7 Jun. 1998, S. Yano leg. (EUMJ); 1 +, same data, 12 Jul. 1987, S. Hisamatsu leg. (EUMJ); 1 ○, Nuno, Tosa-shimizu, 12 Jul. 1987, M. Sakai leg. (EUMJ); 1 ○, Takase, Niyodo-mura, 10 Jul. 2004, Y. Satô leg. (EUMJ); 1 †, Kuroson, Nishitosa-mura, 15-16 Oct.

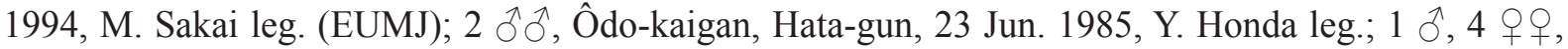
same data, 14 Jul. 2002, M. Sakai leg. (EUMJ); 1 + , Shirahama, Saga-machi, 26 Jul. 1999, J. Ogawa leg. (EUMJ); 1 \&, Nankoku-shi, estuary of Monobe-gawa, 14 Jul. 2002, Y. Kikuhara leg. (EUMJ); 1 ग, Haruno-chô, Niyodogawa-ohashi, 13 Jul. 2002, Y. Kikuhara leg. (EUMJ); 3 ふึર, 1 ㅇ, Tosa-yamada, Odajima, 6 Jul. 2002, T. Kurihara leg. (EUMJ); 2 우, Odajima, Tosa-yamada, Monobe-gawa, 4 Oct. 2002, A. Kitahara leg. (EUMJ); 1 ô, Monobe-gawa, Nishi-machi, Noichi-chô, 1 Jul. 2002, M. and A. Sakai leg. (EUMJ); 1 , same data, 6 Jul. 2002, N. Oshige leg. (EUMJ); 1 $\uparrow, 1$, , Tosa-shi, estuary of Niyodo-gawa, 1 Jul. 2002, M. Sakai leg. (EUMJ); 2 ऊ̂̄, Ino-chô, Okuna, Uji-gawa, 6 Jul. 2002,

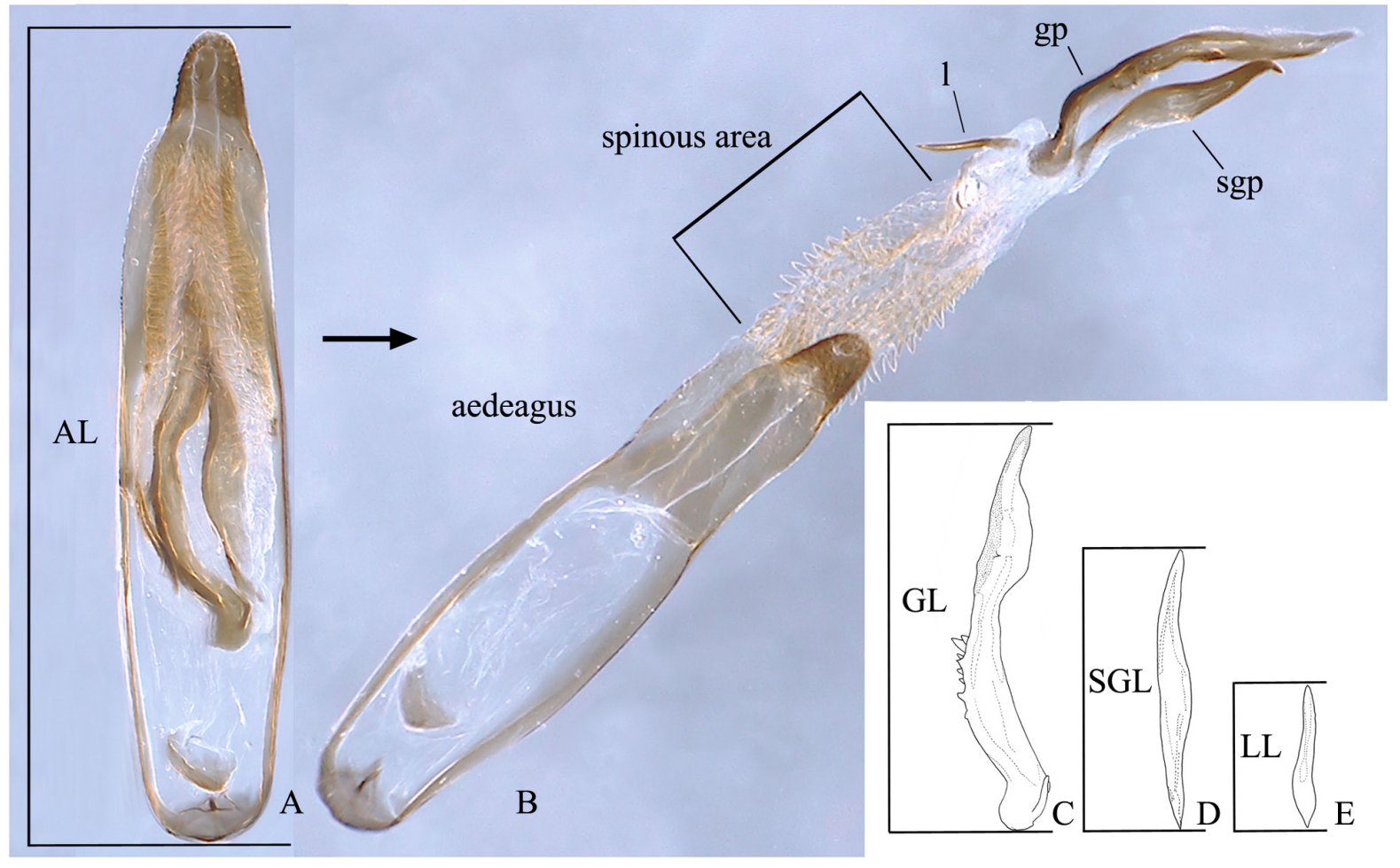

Fig. 7. Aedeagus of Intybia histrio (Kiesenwetter, 1874), showing the terminology and abbreviations for measurement of endophallic structures. A. Aedeagus with holding endophallus. B. Aedeagus with extracted endophallus. C. Gonoporal piece (gp). D. Semi-gonoporal piece (sgp). E. Ligula (l). Abbreviations: $\mathrm{AL}=$ Length of aedeagus; $\mathrm{GL}=$ length of gonoporal piece; $1=$ Ligula; $\mathrm{LL}=$ length of ligula; $\mathrm{SGL}=$ length of semi-gonoporal piece. 
T. Kurihara leg. (EUMJ); 1 đ̊, Ino-chô, Kada-campsite, 16 Jun. 2002, M. and A. Sakai leg. (EUMJ); 1 ô, same data, 14 Jul. 2002, T. Kurihara leg. (EUMJ); 2 సో, Ototake, Ino-chô, 28-29 Jun. 2001, J. Ogawa

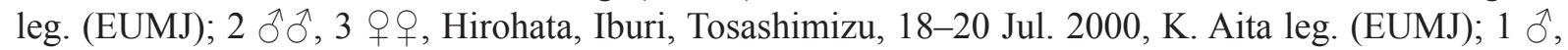
Touno-hama, Yasuda-chô, 29 Jun. 2008, Y. Satô leg. (EUMJ). - Kagawa Pref.: 3 đ̊̄ ô, Konpirasan, 11 Jul. 1991, M. Kawanabe leg. (EUMJ); 1 Õ, 1 क, same data, 12 Jul. 1973, S. Kinoshita leg. (EUMJ); 2 우, same data, 14 Jul. 1973, S. Kinoshita leg. (EUMJ).

JAPAN, Kyushu: Fukuoka Pref.: 1 ð̊, Fukuchi-yama, 15 Jul. 1953, S. Kimoto leg. (EUMJ). - Nagasaki Pref.: 1 \} , 1 \text { q, Hirado-shi, 3-10 Aug. 1974, A. Oda leg. (EUMJ); } 1 \text { q, same data, } 1 \text { Aug. 1975, A. Oda }

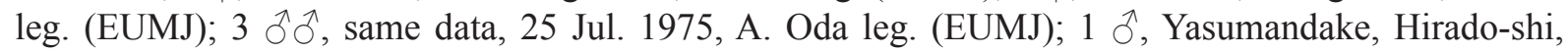
10 Aug. 1979, A. Oda leg. (EUMJ); 1 O, same data, 31 Jul- 2 Aug. 1974, Y. Notsu leg. (EUMJ); 1 o, same data, 7 Aug. 1977, A. Oda leg. (EUMJ); 1 đ, Todoroki-kyo-nr. Taradake, 9 Jul. 1991, M. Kawanabe

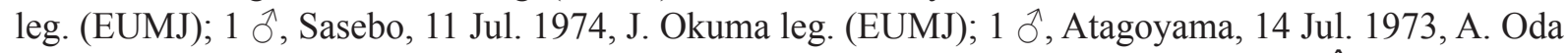
leg. (EUMJ); 1 q, Fugen-san near Isahaya, 20 Jul. 1973, S. Kinoshita leg. (EUMJ); 1 đ̊, Ômura, 10 Aug.

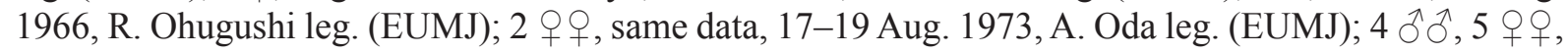
Toishikawa, Hirado-shi, 1 Aug. 1979, A. Oda leg. (EUMJ); 2 đ̂̃ 1 , 1 , Tabira, 8 Aug. 1974, A.Oda leg. (EUMJ); 1 ภ, 2 우, same data, 13 Aug. 1979, A. Oda leg. (EUMJ); 3 $\widehat{\jmath}, 1$ ㅇ, Shikamachi, 30 Jul. 1975, A. Oda leg. (EUMJ). - Oita Pref.: 1 ㅇ, Takashima, 11 Aug. 1953, T. Tachikawa leg. (EUMJ). Kumamoto Pref.: 1 q, Ezu-ko, Kumamoto-shi, 30 Aug. 1991, H. Yoshitomi leg. (EUMJ). - Miyazaki Pref.: 1 ठ, Takamatsu, 3 Jul. 1958, H. Isozaki leg. (EUMJ); 3 q + , Sobo-san, 30 Aug. 1959, H. Isozaki leg. (EUMJ). - Kagoshima Pref.: 4 §ึ ô, Takachiho-mine, Kirishima, 23-24 Jul. 1968, S. Kinoshita leg. (EUMJ); 1 đે, same data, 16 Jul. 1938, H. Araki leg.; 1 ठૈ, Satamisaki, 13 Jul. 1969, M. Sakai leg. (EUMJ); 1 ô, Ohdomari, 14 Jul. 1969, M. Sakai leg. (EUMJ); 1 q, Kimotsuki-chô, Hoyoshi-dake, 19 Jul. 2010, Y. Okahara leg. (EUMJ).

JAPAN, Izu Isls., Hachijô-jima Is.: 1 ㅇ, Hachijô-jima, 23-25 Jul. 2001, N. Ohbayashi leg. (EUMJ).

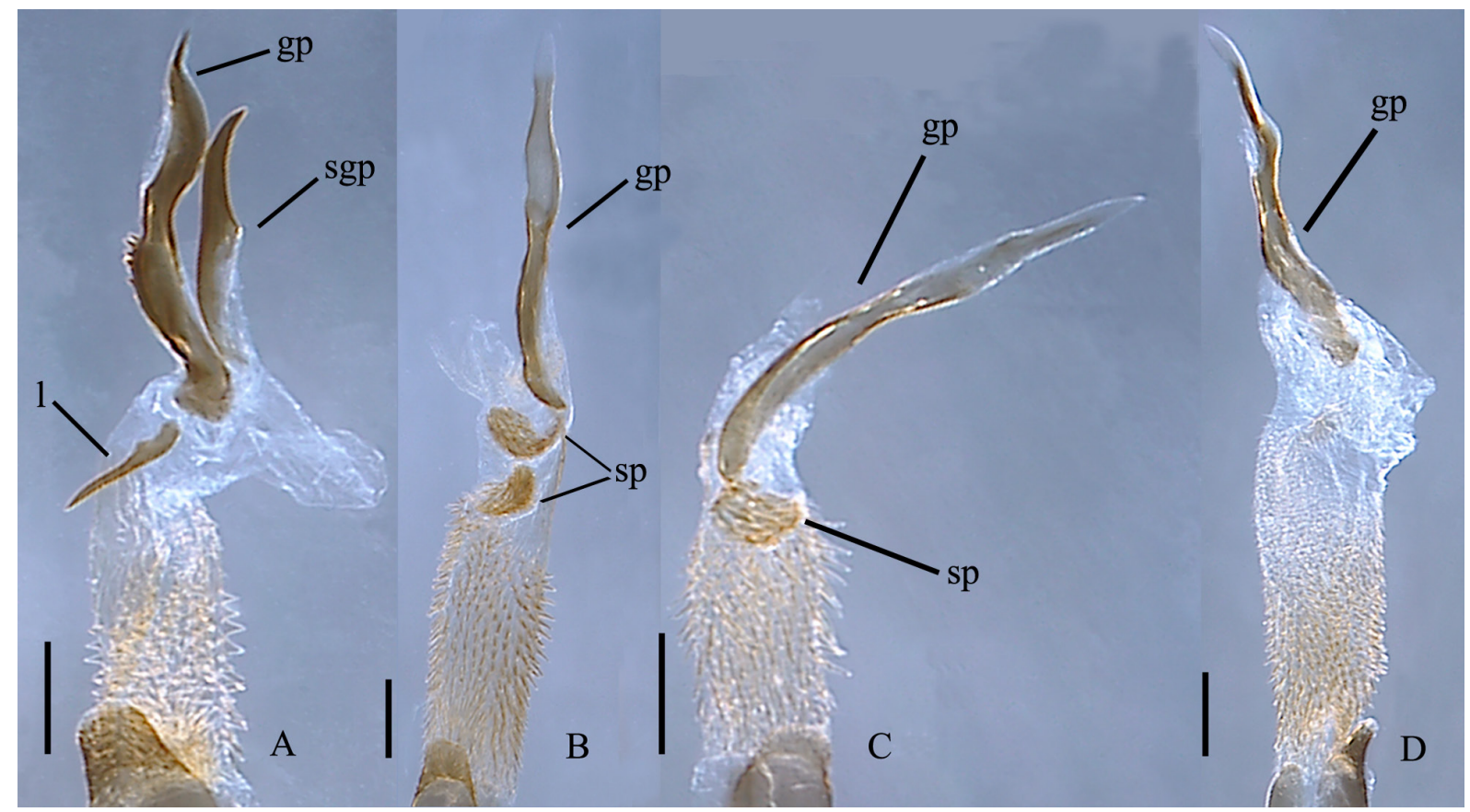

Fig. 8. Endophallus of Intybia spp., A. Intybia histrio (Kiesenwetter, 1874). B. I. pelegrini pelegrini (Pic, 1910). C. I. takaraensis (Nakane, 1955). D. I. kishiii (Nakane, 1955). Abbreviations: gp = gonoporal piece 1 = ligula; $\mathrm{sgp}=$ semi-gonoporal piece; $\mathrm{sp}=$ spinous plate. Scales $=0.1 \mathrm{~mm}$. 
JAPAN, Iki Is.: 1 §, 5 우, Gonoura, 26 Jul. 1977, K. Matsuda leg. (EUMJ).

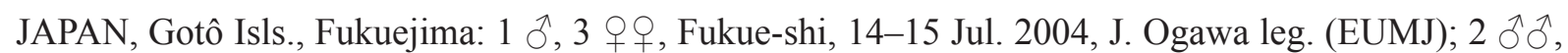
Oritake, 13 Jul. 2008, R. Ogawa leg. (EUMJ); 1 +, Kisyuku-machi, Toginokubi, 12 Jul. 2008, R. Ogawa leg. (EUMJ).

JAPAN, Koshiki Isls., Kamikoshiki-jima: 1 ㅇ, Nakakoshiki, 16-17 Jul. 2002, J. Ogawa leg. (EUMJ).
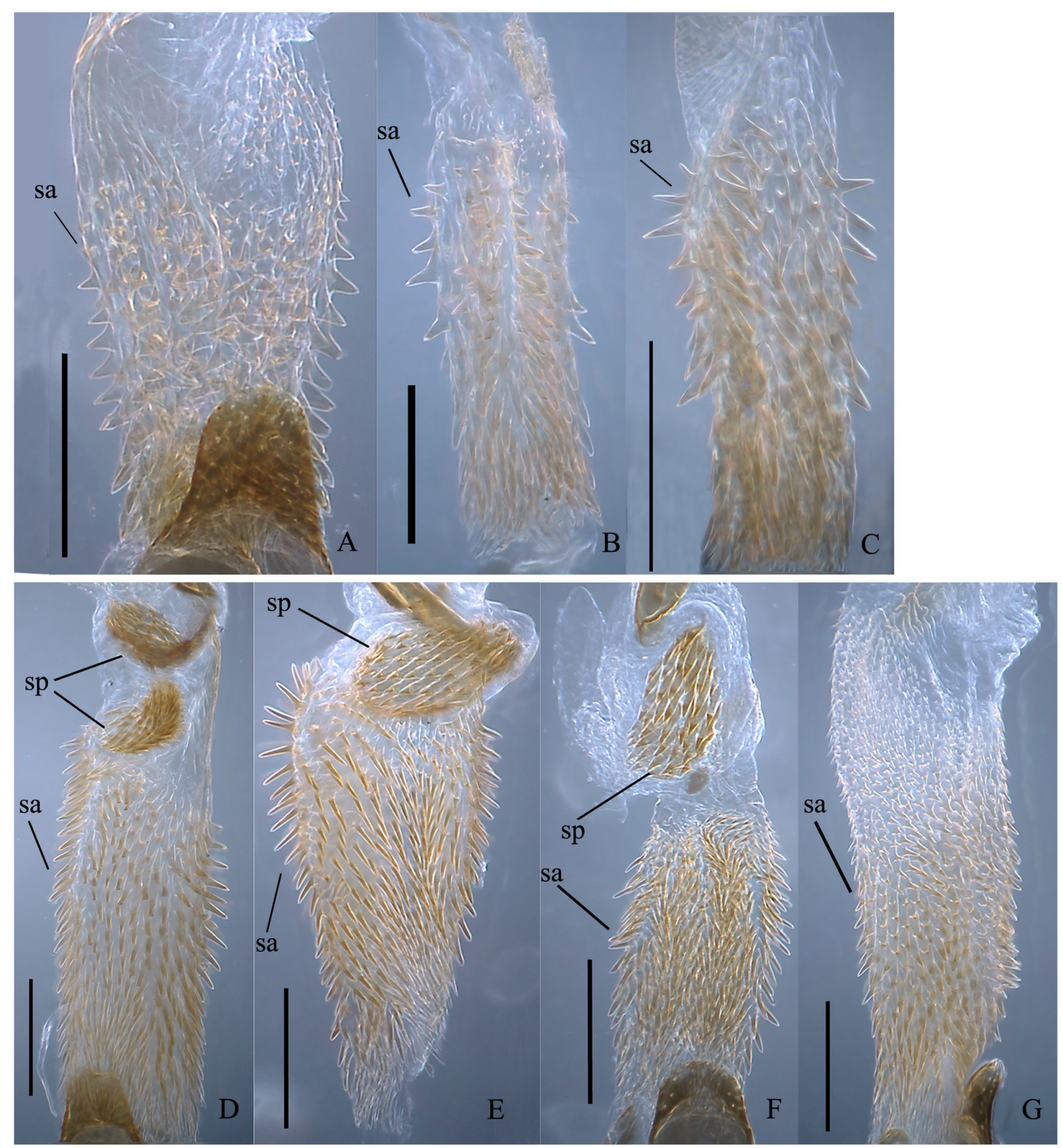

Fig. 9. Spinous area and spinous plate of Intybia spp. A. Intybia histrio (Kiesenwetter, 1874). B. I. tsushimensis (Satô \& Ohbayashi, 1968). C. I. donan sp. nov. D. I. pelegrini pelegrini (Pic, 1910). E. I. niponica (Lewis, 1895). F. I. takaraensis (Nakane, 1955). G. I. kishiii (Nakane, 1955). Abbreviations: $\mathrm{sa}=$ spinous area; $\mathrm{sp}=$ spinous plate. Scales $=0.1 \mathrm{~mm}$. 
JAPAN, Ôsumi Isls., Tanegashima: 1 +, Makinomine, 30 Jul. 1974, H. Makihara leg. (EUMJ). Yakushima: 1 + , North of Kurio, 18 Jul. 1997, H. Yoshitomi leg. (EUMJ); 1 ðૈ, Kurio, 15 Jul. 1970, K. Yamagishi leg. (EUMJ); 1 q, same data, 4 Aug. 1964, T. Okadome leg. (EUMJ).

\section{Redescription}

\section{Male}

Body (Fig. 1A) oblong oval, shining, closely covered with yellow pubescence, sparsely covered with long erect setae. Coloration of head, pronotum and elytra black; antennae yellow; head yellow in apical half; elytra with pale yellow fascia, variable (Fig. 3); legs yellow, but hind femur and tibia black.

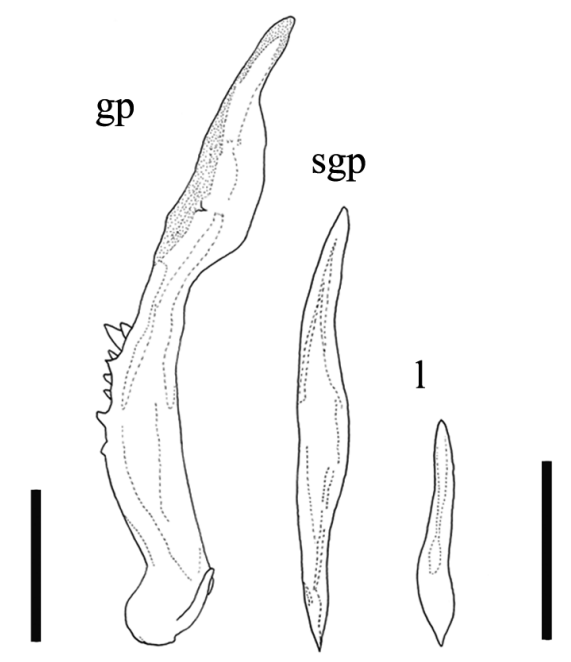

A

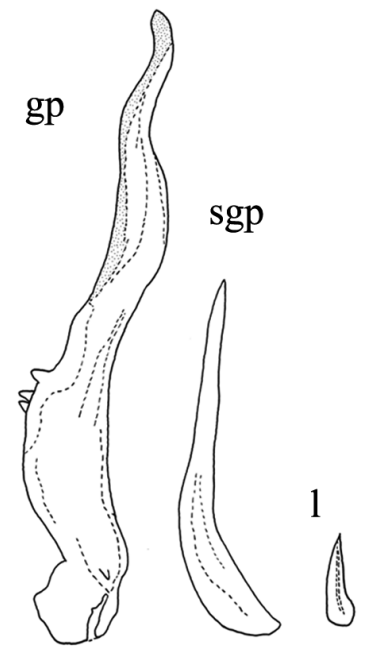

B

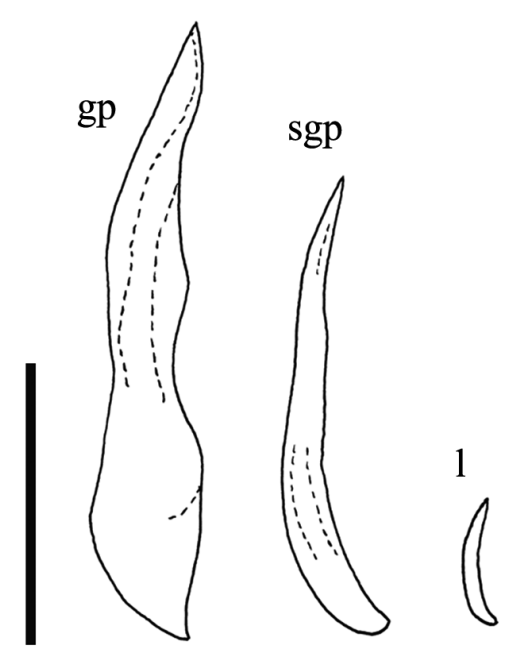

$\mathrm{C}$

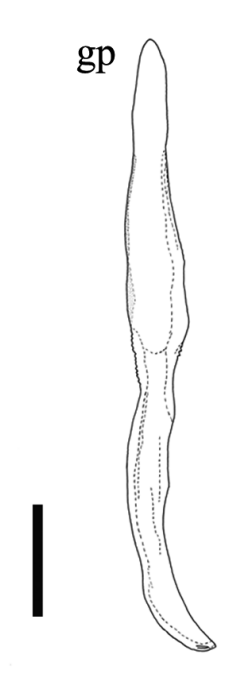

$\mathrm{D}$

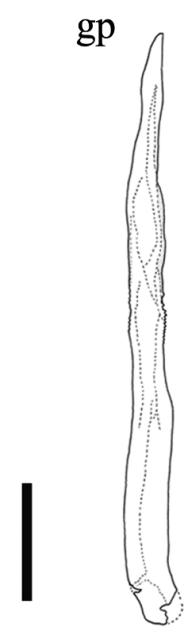

$\mathrm{E}$

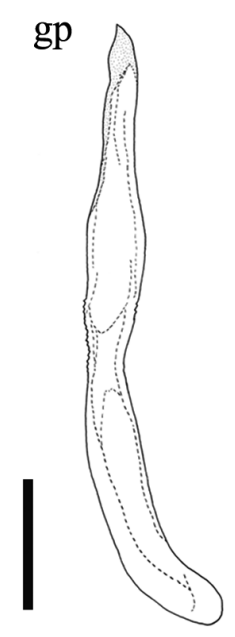

$\mathrm{F}$

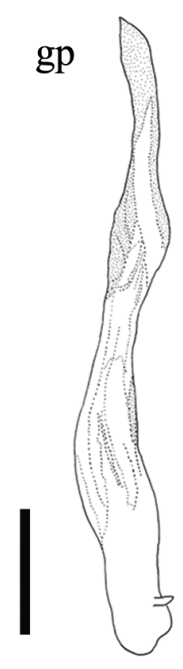

G

Fig. 10. Endophallic sclerites of Intybia spp. A. Intybia histrio (Kiesenwetter, 1874). B. I. tsushimensis (Satô \& Ohbayashi, 1968). C. I. donan sp. nov. D. I. pelegrini pelegrini (Pic, 1910). E. I. niponica (Lewis, 1895). F. I. takaraensis (Nakane, 1955). G. I. kishiii (Nakane, 1955). Abbreviations: gp = gonoporal piece; $1=$ ligula; $\operatorname{sgp}=$ semi-gonoporal piece. Scale bars $=0.1 \mathrm{~mm}$. 
Head sparsely and minutely punctate; eyes normal, moderately prominent; HL/HW 0.73-0.93 (0.84). Antennae (Fig. 4A) long, nearly reaching base of elytra; antennomere I long, abruptly widened from near apical portion; antennomere III (Fig. 6A) fusiform, with a oval funnel-shape on outer edge, providing with a twisted projection near base; $\mathrm{L} / \mathrm{W}=1.76$; approximate ratio of each antennomere $(\mathrm{n}=1)$ as $7.7: 1.0: 7.3: 1.5: 2.0: 2.0: 2.0: 2.2: 2.5: 2.0: 3.0$. Pronotum slightly longer than broader, sparsely and minutely punctate; PW/PL 1.07-1.20 (1.12). Elytra oblong-ovate, slightly broadened posteriorly, shallowly and densely punctate; elytral fascia wide, situated just before the middle, sometimes interrupted by suture; EL/EW 1.38-1.82 (1.49); EL/PL 2.52-2.92 (2.70); EW/PW 1.40-1.77 (1.63); TL/EW 2.282.96 (2.51). Aedeagus (Fig. 7) about $0.84 \mathrm{~mm}$ in AL. Gonoporal piece (Figs 8A, 10A) curved, widened from near the apical portion, with straight basal projection, with serriform process on edge, $0.44 \mathrm{~mm}$ in GL; GL/AL 0.52. Semi-gonoporal piece straight, slender, widened near central part, $0.32 \mathrm{~mm}$ in SGL. Ligula long, straight, $0.16 \mathrm{~mm}$ in LL. Spinous area (Fig. 9A) bearing short triangular spines, becoming smaller in apical portion, lacking spinous plate.

\section{Female}

Similar to male in general appearance (Fig. 2A); head entirely black; HL/HW 0.52-0.68 (0.61); PW/ PL 1.07-1.17 (1.12); EL/EW 1.34-1.78 (1.50); EL/PL 2.80-3.32 (2.95); EW/PW 1.60-1.98 (1.78); TL/EW 2.06-2.64 (2.31). Antennae (Fig. 5A) long; antennomere III distinctly expanded laterally, shallowly concave in dorsal portion; $\mathrm{L} / \mathrm{W}=2.18$; approximate ratio of each antennomere $(\mathrm{n}=1)$ as $6.3: 1.0: 4.0: 2.0: 2.2: 2.2: 2.3: 2.2: 2.2: 2.2: 3.2$.

\section{Measurements}

Male (n = 20): TL 2.86-3.69 (3.38) mm; HL 0.55-0.71 (0.63) mm; HW 0.68-0.81 (0.75) mm; PW 0.73-0.92 (0.83) mm; PL 0.61-0.84 (0.75) mm; EL 1.70-2.23 (2.01) mm; EW 1.12-1.51 (1.35) mm.

Female $(\mathrm{n}=20)$ : TL 3.19-3.94 (3.52) mm; HL 0.40-0.58 (0.48) mm; HW 0.73-0.90 (0.79) mm; PW 0.77-0.97 (0.86) mm; PL 0.7-0.85 (0.77) mm; EL 2.05-2.51 (2.27) mm; EW 1.32-1.72 (1.53) mm.

\section{Distribution (Fig. 11A)}

Japan (Honshu, Shikoku, Kyushu, Hachijô-jima [NR], Iki Is., Fukue-jima, Tanega-shima [NR], Yakushima); Russia (Kunashir Is.), China (Shanghai).

\section{Remarks}

This species closely resembles Intybia tsushimensis (Satô \& Ohbayashi, 1968), and is distinguished from the latter by the coloration and structure of the endophallus. These species are distributed allopatrically.

\section{Biological notes}

This species is the most common in mainland Japan. It inhabits forest edges and grasslands (Fig. 12A), and can be collected from shrubs and bushes by sweeping and beating.

Intybia tsushimensis (Satô \& Ohbayashi, 1968)

[Japanese name: Tsushima-hiro-obi-jôkai-modoki]

Figs 1B, 2B, 4B, 5B, 6B, 9B, 10B, 11A

Laius tsushimensis Satô \& Ohbayashi, 1968: 5 [type locality: Mt. Ariake-yama, Tsushima].

Laius tsushimensis - Nakane 1983: 164.—Satô 1985: 164, pl. 26, fig. 6.—Satô 1989: 366.

Intybia tsushimensis - Wittmer 1997: 191.- Mayor 2007: 417 [catalogued].- Yoshitomi \& Hayashi 2011: 19. 


\section{Diagnosis}

Elytra with a pale yellow fascia, just before the middle, which is narrowed at suture; legs fully black; antennomere III cylinder-like shaped, shallowly concave in dorso-mesal portion, and provided with a tongue-like projection near behind; gonoporal piece pluri-curved, slender; semi-gonoporal piece slightly curved; ligula short; spinous plate absent.

\section{Material examined}

\section{Holotype}

JAPAN: Ô, [Is. Tsushima, Mt. Ariake, July, 16. 1960, M. Satô leg.] (EUMJ).

\section{Paratypes}

JAPAN: $1 \stackrel{\lambda}{\partial} 1$, same data as for the holotype; 1 , Izuhara, Tsushima, 21 Aug. 1968, S. Nomoto leg. (EUMJ).

\section{Additional specimens}

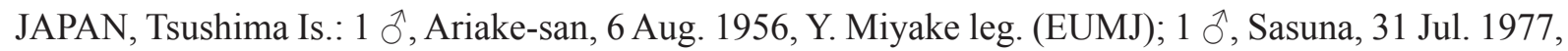
K. Matsuda leg. (EUMJ); 1 , same data, 2 Aug. 1977, K. Matsuda leg. (EUMJ); 1 , Mitake, 29 Jun. 1980, N. Ohbayashi leg. (EUMJ); 1 §, Sasuna, 14 Jul. 1985, K. Ando leg. (EUMJ); 1 ð,, Mokkoku-san,

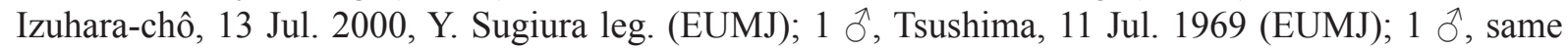
data, 14 Jul. 1969, S. Miyamato leg. (EUMJ); 1 q, Izuhara-Sasutôge, 24 Aug. 1941, T. Shirôzu leg. (SEHU); $2 \lesssim \precsim$, Shitasaki, Kamitsuhisma-machi, 21 Jun. 2002, T. Kurihara leg. (EUMJ); 1 ठ, Waniura, Kamitsushima-machi, 21 Jun. 2002, T. Kurihara leg. (EUMJ); 1 ð̊, Kechi, Mitsushima-chô, 22 Jun. 2002, T. Kurihara leg. (EUMJ); 1 đ̃, Sumo, Mitsushima-chô, 15 Jun. 2002, T. Kurihara leg. (EUMJ); 1 , Ayumodoshi-shizenkoen, Izuhara-machi, Tsushima-shi, 8 Aug. 2013, N. Tsuji leg. (NTJ).

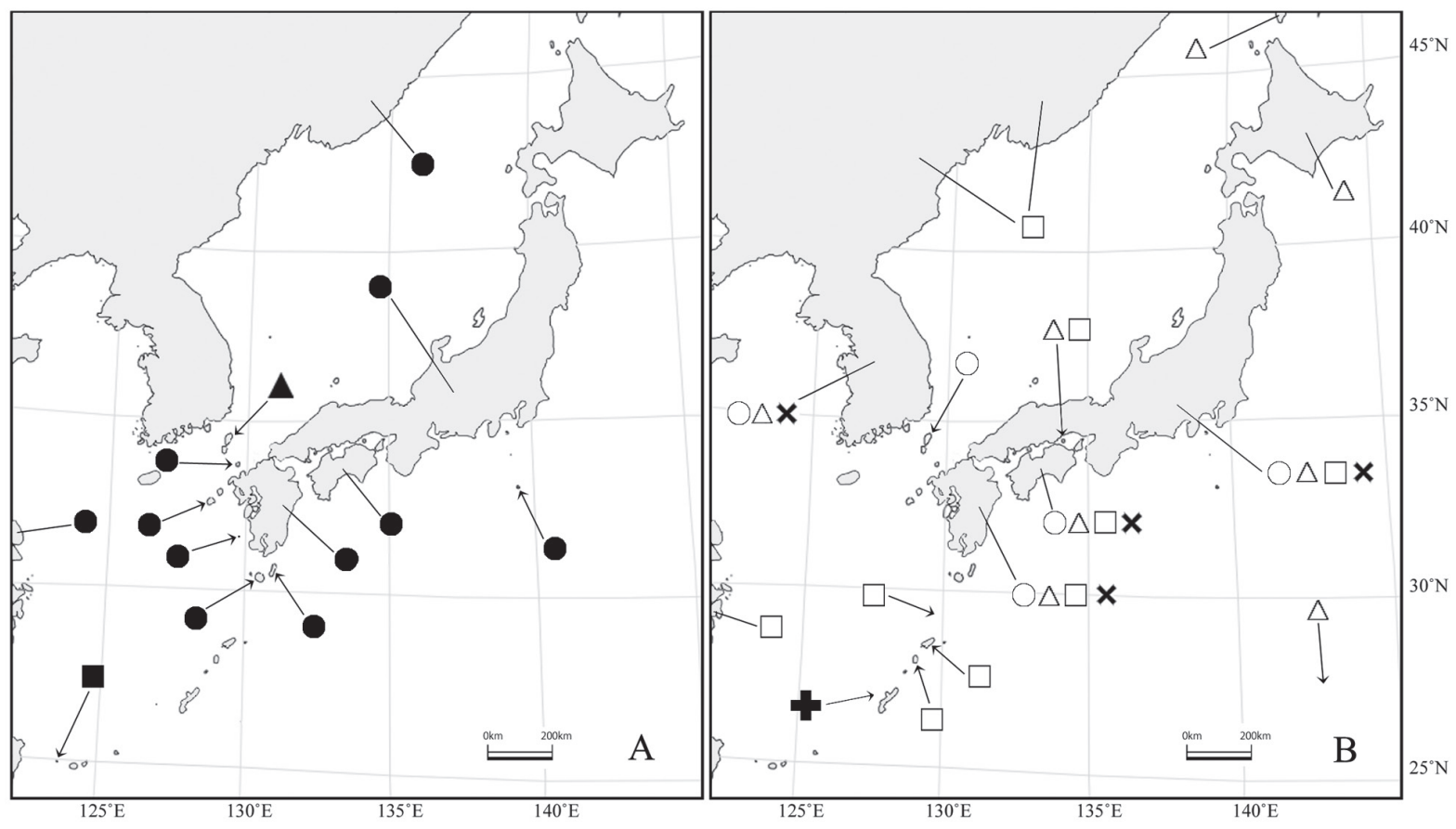

Fig. 11. Map showing the distribution of Intybia spp. A. The histrio group: $\bullet$ : Intybia histrio (Kiesenwetter, 1874), $\mathbf{\Delta}$ : I. tsushimensis (Satô \& Ohbayashi, 1968), a: I. donan sp. nov.). B. The pelegrini group: $\circ$ : I. pelegrini (Pic, 1910), $\triangle$ : I. niponica (Lewis, 1895), $\square:$ I. takaraensis (Nakane, 1955), $\times$ : I. kishiii (Nakane, 1955), +: I. kawasakii (Nakane, 1956). 


\section{Redescription}

\section{Male}

Body (Fig. 1B) oblong oval, shining, densely covered with yellow pubescence. Coloration of head, pronotum and elytra black; antennae yellow, with apical 6 or 7 segments black; elytral fascia pale yellow; legs black.

Head sparsely and minutely punctate; eyes normal, moderately prominent; HL/HW 0.59-0.89 (0.71). Antennae (Fig. 4B) long, nearly reaching base of elytra; antennomere III (Fig. 6B) cylinder-like shaped, shallowly concave in dorso-mesal portion, with a tongue-like projection posteriorly; $\mathrm{L} / \mathrm{W}=1.47$; approximate ratio of each antennomere $(n=1)$ as $13.0: 1.0: 14.7: 2.7: 3.3: 3.7: 4.0: 4.0: 4.0: 3.7: 5.0$. Pronotum slightly broader than long, sparsely and minutely punctate; PW/PL 1.06-1.17 (1.14). Elytra oblong-ovate, slightly broadened posteriorly, shallowly and somewhat densely punctate; elytral fascia situated just before the middle, narrowed at suture; EL/EW 1.27-1.71 (1.49); EL/PL 2.35-2.94 (2.80); EW/PW 1.53-1.83 (1.69); TL/EW 2.17-2.72 (2.41). Aedeagus about $1.00 \mathrm{~mm}$ in AL. Gonoporal piece (Fig. 10B) winding, slender, bearing minute spines in middle part, $0.32 \mathrm{~mm}$ in GL; GL/AL 0.32. Semi-
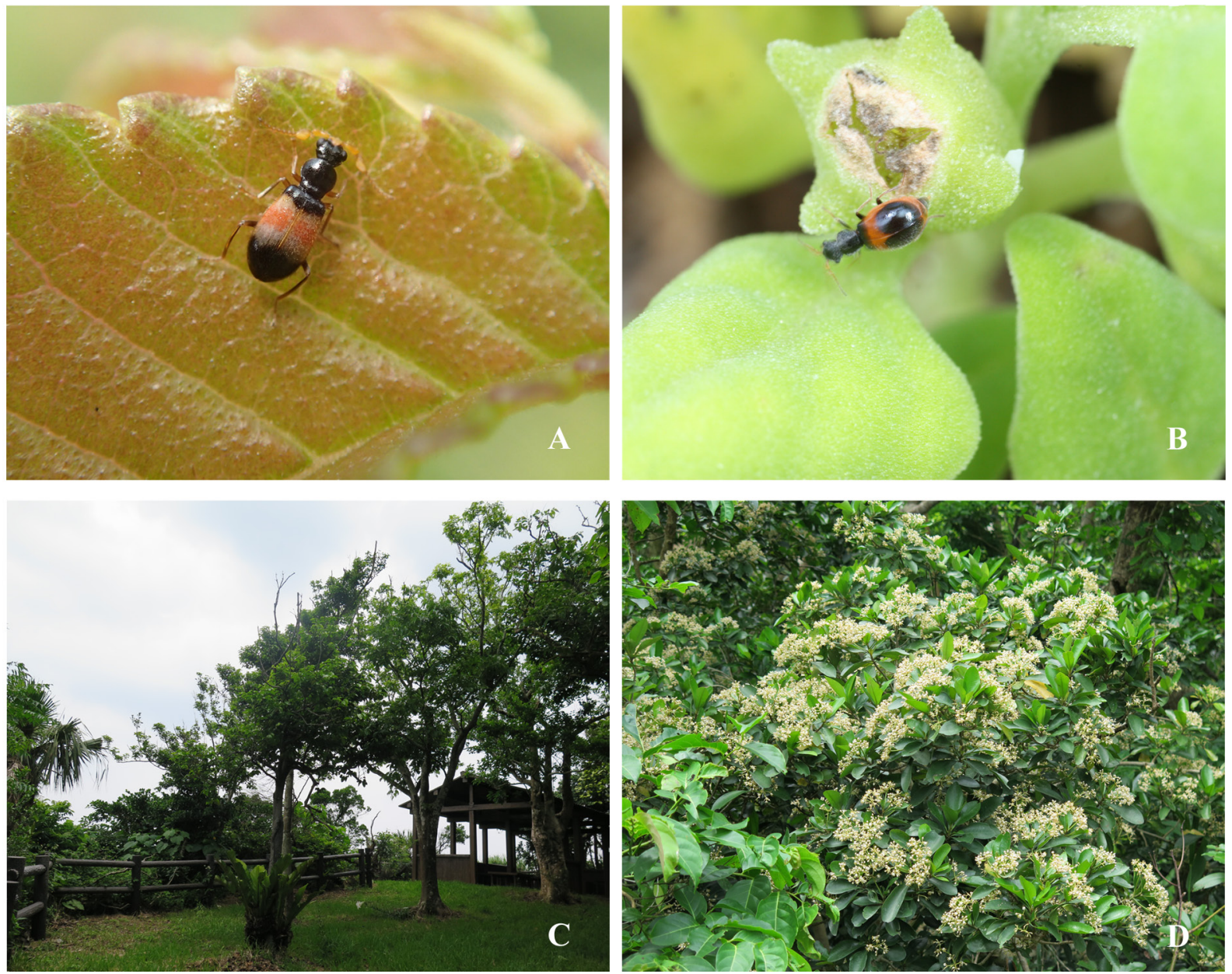

Fig. 12. Habitat and habits of Intybia spp. A. Intybia histrio, đo (at Awa-shima, Kagawa Pref.). B. I. niponica, + (at Saijo-shi, Ehime Pref.), photographs by HY. C - D. Type locality of I. donan sp. nov. (Mantabaru-shinrinkôen, Yonaguni-chô, Yaeyama-gun, Okinawa Pref.), showing the flower of Ardisia sieboldii (D), photographs by HI. 
gonoporal piece slightly curved, $0.20 \mathrm{~mm}$ in SGL. Ligula short, $0.05 \mathrm{~mm}$ in LL. Spinous area (Fig. 9B) bearing short triangular spines, lacking spinous plate.

\section{Female}

Similar to male in general appearance (Fig. 2B); HL/HW 0.57-0.79 (0.67); PW/PL 1.03-1.21 (1.14); EL/EW 1.36-1.71 (1.52); EL/PL 2.76-3.19 (3.01); EW/PW 1.51-1.87 (1.74); TL/ EW 2.11-2.68 (2.40). Antennae long (Fig. 5B); antennomere III distinctly expanded laterally, shallowly concave in dorsal portion, $\mathrm{L} / \mathrm{W}=2.30$; approximate ratio of each antennomere $(\mathrm{n}=1)$ as $9.7: 1.0: 7.7: 3.7: 3.7: 3.7: 4.0: 4.0: 4.0: 3.3: 4.0$.

\section{Measurements}

Male (n = 12): TL 3.07-3.64 (3.39) mm; HL 0.47-0.63 (0.55) mm; HW 0.57-0.87 (0.78) mm; PW 0.75-0.88 (0.84) mm; PL 0.67-0.81 (0.75) mm; EL 1.90-2.32 (2.09) mm; EW 1.19-1.54 (1.41) mm.

Female $(\mathrm{n}=6)$ : TL 3.38-4.05 (3.58) mm; HL 0.50-0.70 (0.56) mm; HW 0.77-0.89 (0.83) mm; PW 0.79-0.95 (0.86) mm; PL 0.70-0.80 (0.75) mm; EL 2.10-2.55 (2.25) mm; EW 1.28-1.74 (1.49) mm.

Distribution (Fig. 11A)

Japan (Tsushima Is.)

\section{Remarks}

The species is similar to Intybia histrio (Kiesenwetter, 1874) in the shape of the male antennomere III, elytral coloration and endophallic structures, and differs from it in the coloration of the legs and antennae, and in the shapes of the semi-gonoporal piece and ligula. This species seems to be endemic to Tsushima Is.

\section{Biological notes}

This species inhabits forest edges and grasslands, and can be collected from shrubs and bushes by sweeping and beating.

$$
\begin{gathered}
\text { Intybia donan sp. nov. } \\
\text { urn:1sid:zoobank.org:act:DCD7FF92-1188-40E9-8AE9-94BE4CA028B7 } \\
\text { [Japanese name: Yotsu-mon-obi-jôkai-modoki] } \\
\text { Figs 1C, 2C, 4C, 5C, 6C, H, 9C, 10C, 11A, 12C-D }
\end{gathered}
$$

\section{Diagnosis}

Elytra with four pale yellow spots; antennomere I long, abruptly widened from near the middle; antennomere III ginkgo-like shaped, two concavities on outer edge and provided with a slender tonguelike projection near base; gonoporal piece gently curved, constricted near the middle; semi-gonoporal piece slightly curved; ligula long, slightly curved; spinous plate absent.

\section{Etymology}

After the old name of the type locality Yonaguni-jima.

\section{Material examined}

\section{Holotype}

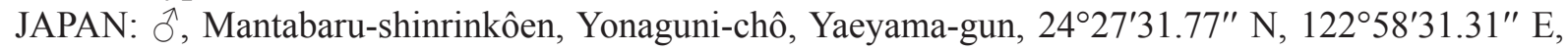
4 May 2016, H. Ikeda leg. (EUMJ). 


\section{Paratypes}

JAPAN: $2 \hat{\partial} \hat{\partial}, 1$, same data as for the holotype (EUMJ); $3 \hat{\partial}$, same data as for the holotype, but 30 Apr. 2016, N. Tsuji leg. (NTJ); 2 우, same data as for the holotype, but 30 Apr. 2016, H. Ikeda leg.

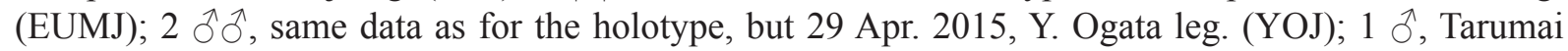
(LT), Yonaguni-jima, 10 Sep. 2004, Shiho and Koji Arai leg. (EUMJ); 1 ô, Yonaguni-jima, Urabu-dake, 28 Apr. 1993, Y. Kaneko leg. (EUMJ); 1 †, Yonaguni-jima, Tokorono, 13 May 1974, H. Hiramatsu leg. (KUM).

\section{Description}

\section{Male}

Body (Fig. 1C) oblong oval, shining, densely covered with yellow pubescence, and sparsely covered with long erect hairs. Coloration of head, pronotum and elytra black; antennae, two pairs of elytral spots, and legs pale yellow.

Head sparsely and minutely punctate; eyes moderately prominent; HL/HW 0.51-0.79 (0.63). Antennae (Fig. 4C) long, nearly reaching base of elytra; antennomere I long, abruptly widening from middle onwards; antennomere III (Fig. 6C) ginkgo-leaf shaped, with two concavities on outer edge, with a slender tongue-like projection near base; $\mathrm{L} / \mathrm{W}=1.53$; approximate ratio of each antennomeres $(\mathrm{n}=1)$ as $8.3: 1.0: 9.7: 2.3: 2.7: 3.3: 3.3: 3.3: 3.3: 3.7: 6.3$. Pronotum slightly broader than long, sparsely and minutely punctate; PW/PL 1.07-1.27 (1.18). Elytra oblong-ovate, slightly broadened posteriorly, somewhat densely punctate; two large and oval pairs of elytral spots; EL/EW 1.22-1.48 (1.39); EL/PL 2.58-3.14 (2.77); EW/PW 1.48-1.82 (1.69); TL/EW 2.02-2.56 (2.27). Aedeagus about $0.48 \mathrm{~mm}$ in AL. Gonoporal piece (Fig. 10C) gently curved, constricted from near the middle $0.23 \mathrm{~mm}$ in GL; GL/AL 0.48 . Semi-gonoporal piece slightly curved, $0.19 \mathrm{~mm}$ in SGL. Ligula long slightly curved, $0.05 \mathrm{~mm}$ in LL. Spinous area (Fig. 9C) bearing short spines, lacking spinous plate.

\section{Female}

Similar to male in general appearance (Fig. 2C); HL/HW 0.52-0.60 (0.57); PW/PL 1.08-1.18 (1.15); EL/EW 1.34-1.43 (1.40); EL/PL 2.73-3.04 (2.94); EW/PW 1.78-1.92 (1.83); TL/ EW 2.08-2.23 (2.18). Antennae (Fig. 5C) long; antennomere III distinctly expanded laterally, shallowly concave in dorsal portion, $\mathrm{L} / \mathrm{W}=1.78$; approximate ratio of each antennomere $(\mathrm{n}=1)$ as $6.3: 1.0: 4.0: 2.3: 2.5: 2.5: 2.8: 2.8: 2.8: 2.8: 4.8$.

\section{Measurements}

Male $(\mathrm{n}=10)$ : TL 2.23-2.52 (2.40) mm; HL 0.32-0.46 (0.39) mm; HW 0.57-0.67 (0.62) mm; PW 0.60-0.67 (0.63) mm; PL 0.50-0.58 (0.53) mm; EL 1.31-1.62 (1.48) mm; EW 0.89-1.20 (1.06) mm.

Female (n = 4): TL 2.46-2.88 (2.66) mm; HL 0.32-0.42 (0.37) mm; HW 0.62-0.70 (0.66) mm; PW $0.62-0.73$ (0.67) mm; PL 0.53-0.62 (0.58) mm; EL 1.61-1.84 (1.70) mm; EW 1.12-1.30 (1.22) mm.

\section{Distribution (Fig. 11A)}

Only known from the type locality, Yonaguni-jima of Japan.

\section{Remarks}

This species is distinct in having a small body and four elytral spots. It is similar to Intybia viridithorax (Pic, 1921) in the shapes of antenna and male genitalia (Wittmer 1997), and differs from the latter by the coloration of the elytra with four spots (I. viridithorax has fascia). 


\title{
Biological notes
}

This species is rare, inhabits forest edges (Fig. 12C), can be collected from the flowers of Ardisia sieboldii Miquel and Meliosma oldhamii Miquel ex Maximowicz (Fig. 12D), and can be collected from shrubs and bushes by sweeping, beating, and light traps.

\author{
Intybia pelegrini pelegrini (Pic, 1910) \\ [Japanese name: Kiashi-obi-jôkai-modoki] \\ Figs 1D, 2D, 4D, 5D, 6D, 8D, 9D, 10D, 11B
}

Laius pelegrini Pic, 1910: 62 [type locality: Yedo, Japon].

Laius pelegrini-Champion 1921: 331.—Pic 1921: 19._- Nakane 1983: 164.

Intybia pelegrini pelegrini - Plonski 2013: 66.

Laius pellegrini [misspelling] - Greiner 1937: 152. — Nakane 1957: 13, fig. 2. — Nakane 1963: 186, pl. 93, fig.14. — Satô 1985: 165, pl. 26, fig. 10. — Satô 1989: 366.

Intybia pellegrini pellegrini [misspelling]: Wittmer 1997: 181, figs 34-36. - Mayor 2007: 417 [catalogued]. - Yoshitomi \& Hayashi 2011: 19.

\section{Diagnosis}

Elytra with a wide yellow fascia just before the middle, which is widened on lateral margin, and indented posteriorly; antennomere III half crescent-like shaped, excavate on upper surface; gonoporal piece straight, slender, slightly curved basally; semi-gonoporal piece and ligula absent, with two spinous plates.

\section{Material examined}

JAPAN, Honshu: Fukushima Pref.: 1 \% , Abukuma-gawa, 22 Aug. 1980, S. Ohmomo leg. (EUMJ). Tochigi Pref.: 1 đ̃, Tochigi-shi, Watarase-yusuichi, 22 Sep. 2014, H. Suenaga leg. (HSJ). - Chiba Pref.: 1 , Narashino, 13 Jun. 1951, H. Hattori leg. (EUMJ). - Saitama Pref.: 3 q $ᄋ$, Toda-shi, Niizo, 19 Jun. 1986, M. Hasegawa leg. (EUMJ). - Kanagawa Pref.: 1 q, Tsukuihama, 2 Aug. 1985, N. Ohbayashi leg. (EUMJ). - Niigata Pref.: 2 ㅇ, , Echigo, Miomote, 20 Jul. 1959, K. Baba leg. (EUMJ). - Shizuoka Pref.: 1 , estuary of Fuji-kawa, Kambara-gun, Fukiageno-hama, 31 Aug. 2008, Y. Satô leg. (EUMJ). - Aichi Pref.: 1 Ĵ, (already reported by Wittmer 1997), Chita-shi, Minamihama-chô, 15 Jul. 1982, B. Tanaka

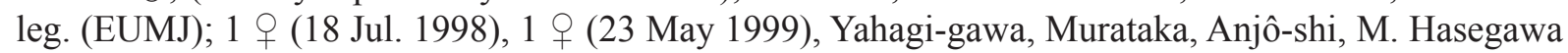
leg. (EUMJ). - Gifu Pref.: 2 우, Tokuyama Dam, 18 Jul. 1992, M. Hibino leg. (EUMJ). - Okayama Pref.: 1 ô, Hyakken-gawa, Okayama-shi, Fujiwara-chô, 29 Sep. 2013, H. Suenaga leg. (HSJ).

JAPAN, Shikoku: Ehime Pref.: $1 \hat{\jmath}$ (misidentified and reported as Lajus niponicus by Miyatake \& Kobayashi 1950), Omogokei, 24 Jul. 1947, M. Miyatake leg. (EUMJ); 1 § (already recorded by Yano 1957), Onogahara, Higashiuwa, 4 Aug. 1955, Y. Wake leg. (EUMJ); 2 $\widehat{\jmath}, 1$ q, Seiyo-shi, Uwa-chô, Yamada, 19 Jun. 2004, T. Kurihara et al. leg. (EUMJ); 1 ò, Zenoji, Matsuyam-shi, Shin-ike, 7 Jun. 2006, J. Ogawa leg. (EUMJ); 1 đૈ, Onishi-chô, Shin-chô, Imabari-shi, Kojin-ike, 6 Jul. 2013, R. Okano

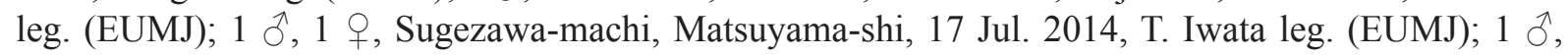

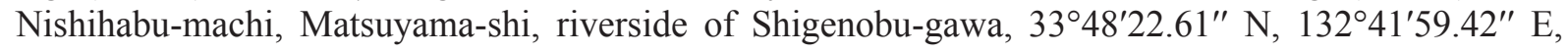
4 Sep. 2015, H. Ikeda leg. (EUMJ); 1 \&, Tanouwa-gata, Tanbara-chô, Saijô-shi, 15 Jul. 2010, S. Matsuo leg. (EUMJ). - Kochi Pref.: 2 qo , Okuna, Ino-chô, Uzi-gawa, 6 Jul. 2002, T. Kurihara leg. (EUMJ); 2 우, Nishimachi, Noichi-chô, 13 Jul. 2002, M. Sakai leg. (EUMJ).

JAPAN, Tsushima Is.: 1 + (already reported by Satô \& Ohbayashi (1968)), Izuhara, 21 Aug. 1966, S. Nomoto leg. (EUMJ); 1 ㅇ, Kechi Dam, 29 Jul. 1979, M. Kotani leg. (EUMJ). 


\section{Redescription}

Male

Body (Fig. 1D) slightly elongate, with gray pubescence. Coloration of head, antennomeres I-III, pronotum, and elytra black; elytral fascia and legs yellow.

Head densely punctate, rugosely so in basal part; eyes oval, moderately prominent; HL/HW 0.65-0.84 (0.72). Antennae (Fig. 4D) long, reaching base of elytra; antennomere III (Fig 6D) half crescent-like shaped, deeply concave in dorso-mesal part; $\mathrm{L} / \mathrm{W}=1.49$; approximate ratio of each antennomere $(\mathrm{n}=1)$ as $5.8: 1.0: 11.2: 1.7: 1.8: 2.0: 2.2: 2.2: 2.5: 2.2: 2.8$. Pronotum rather densely punctate in lateral parts; PW/PL 0.97-1.09 (1.04). Elytra oblong-ovate, slightly broadened posteriorly, densely punctured; elytral fascia wide, situated just before the middle, widened at suture and lateral margins; EL/EW 1.27-1.69 (1.50); EL/PL 2.55-2.79 (2.65); EW/PW 1.46-2.05 (1.70); TL/EW 2.07-2.79 (2.46). Aedeagus about $1.00 \mathrm{~mm}$ in AL. Gonoporal piece (Figs. 8B, 10D) straight, slender, slightly curved basally, $0.52 \mathrm{~mm}$ in GL; GL/AL 0.52. Semi-gonoporal piece and ligula absent. Spinous area (Fig. 9D) densely covered with long spines, with two small spinous plates.

\section{Female}

Similar to male in general appearance (Fig. 2D); coloration of antennae yellow, with apical 6 or 7 segments black; HL/HW 0.59-0.85 (0.68); PW/PL 0.96-1.11 (1.04); EL/EW 1.36-1.66 (1.53); EL/ PL 2.5-2.84 (2.68); EW/PW 1.55-1.89 (1.69); TL/EW 2.14-2.79 (2.48). Antennae (Fig. 5D) long; antennomere III oblong, distinctly expanded laterally, shallowly concave in dorsal portion; $\mathrm{L} / \mathrm{W}=2.9$; approximate ratio of each antennomere $(\mathrm{n}=1)$ as $6.2: 1.0: 5.8: 2.4: 2.4: 2.2: 2.2: 2.4: 2.4: 2.4: 3.6$.

\section{Measurements}

Male (n = 11): TL 3.29-3.93 (3.58) mm; HL 0.54-0.69 (0.58) mm; HW 0.75-0.86 (0.80) mm; PW 0.80-0.94 (0.86) mm; PL 0.77-0.90 (0.82) mm; EL 1.97-2.39 (2.18) mm; EW 1.27-1.64 (1.46) mm.

Female (n = 18): TL 3.58-4.18 (3.88) mm; HL 0.50-0.72 (0.59) mm; HW 0.80-0.92 (0.87) mm; PW 0.82-1.00 (0.93) mm; PL 0.82-1.00 (0.89) mm; EL 2.20-2.56 (2.39) mm; EW 1.40-1.87 (1.57) mm.

Distribution (Fig. 11B)

Japan (Honshu, Shikoku, Kyushu, Tsushima); Russia (Kunashir Is.).

\section{Remarks}

This species is distinct in having a slender body shape, a characteristic coloration, a special shape of the male antennomere III, and two spinous plates.

Wittmer (1997) described the subspecies, I. pelegrini longior Wittmer, 1997, for the Korean population, and this subspecies differs from the nominotypical subspecies (= Japanese population) by the male antennomere III more elongate by $15-20 \%$ (Wittmer 1997).

\section{Biological notes}

This species inhabits grassland areas near large rivers.

Intybia niponica (Lewis, 1895)

[Japanese name: Kuro-ki-obi-jôkai-modoki]

Figs 1E, 2E, 4E, 5E, 6E, 9E, 10E, 11B, 12B

Laius niponicus Lewis, 1895: 116 [type locality: Hakodate sand-hills, also at Kobé]. 
Laius niponicus - Champion 1921: 332. — Greiner 1937: 152. — Nakane 1957: 13, fig. 3. — Nakane 1963: 186, pl. 93, fig. 15. — Nakane 1983: 164. — Satô 1985: 164, pl. 26, fig. 8. — Satô 1989: 366. Intybia niponica - Satô et al. 2006: 349.

Intybia niponicus - Wittmer 1997: 191. — Mayor 2007: 417 [catalogued]. — Yoshitomi \& Hayashi 2011: 19. - Asano 2013: 21.

\section{Diagnosis}

Elytra with a yellow fascia, widened at suture and lateral margins; antennomere III broad bean-like shape excavated on dorsal surface; gonoporal piece straight, slender; semi-gonoporal piece and ligula absent; one spinous plate present.

\section{Material examined}

JAPAN, Hokkaido: 1 ô, 5 q , Wakkanai, 7 Jul. 1977, K. Baba leg. (EUMJ); 1 ô, Shakotan, 22 Jun. 1986, M. Satô leg. (EUMJ); 1 đ̃, Toyotomi, Toyotoku, 20 Jun. 1961, Y. Nishijima leg. (SEHU); 1 ðૈ, Teine, 25 Oct. 1961, K. Kamimura leg. (SEHU); 1 đ, Kushiro-shi, Otanoshike, 1 Jul. 2012, H. Suenaga leg. (HSJ); 1 §̂, Nanaehama, Hakodate-shi, 17 Aug. 1977, N. Nishikawa leg. (EUMJ); 1 q, Ishikarihama, 22 Aug, S. Takagi leg. (SEHU); 1 \%, Oshima, Oshamanbe, 15 Jun. 1956, Y. Nishijima leg. (SEHU); 1 +, same data, 12 Aug. 2009, M. Ôhara leg. (SEHU); 1 †, Ishikari-shi, Atsuta-ku, Shippu, H. Suenaga leg. (HSJ).

JAPAN, Honshu: Yamagata Pref.: 2 $\widehat{\jmath}, 1$ ㅇ, Nishiarase, 28 May 1953, K. Shirahata leg. (EUMJ). Niigata Pref.: 1 ô, Kurokawa, Echigo, 11 Jul. 1964, K. Baba leg. (EUMJ). - Chiba Pref.: 1 q, Shirahamachô, 22 Jun. 2001, T. Ito leg. (EUMJ). - Shizuoka Pref.: 1 q, Kambara-gun, Fukiagenohama, Estualy of Fujikawa, 31 Aug. 2008, Y. Satô leg. (EUMJ). - Aichi Pref.: 6 우어, Omotehama, Toyohashi, 24 Jun. 1990, M. Satô leg. (EUMJ).

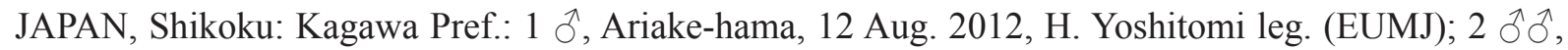
3 우 옹 same data, 15 Aug. 2015, H. Yoshitomi leg. (EUMJ); 1 ㅇ, same data, 28 Jun. 2015, H. Yoshitomi

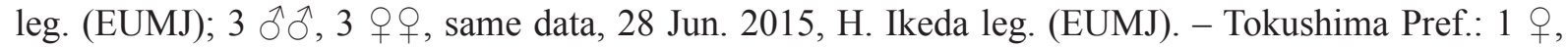

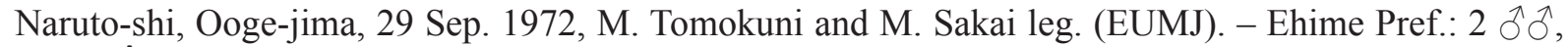
8 우, Ômishima, 19 Jul. 1959, M. Satô leg. (EUMJ); $2 \widehat{\partial}^{\lambda}, 5$ 우 , Odagahama, Higashimura, Imabarishi, 29 Aug. 2000, J. Ogawa leg. (EUMJ); 5 ふふ, 1 ㅇ, Sakurai, Imabari-shi, 10 Jun. 2012, H. Yoshitomi

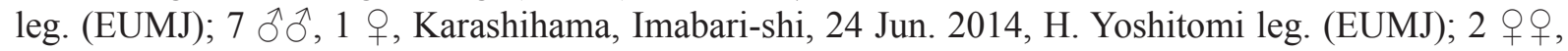
Ohkushi-hama, Nakajima, Matsuyama-shi, 6 Jul. 2010, S-T. Hisamatsu leg. (EUMJ); 1 ô, Matsuyama, 5 Jun. 1973, K. Ito leg. (EUMJ); 1 \& (already reported by Yano 1957), Matsuyama, Yoshida-hama, 31 Aug. 1950, M. Miyatake leg. (EUMJ); 2 우, same data, 31 Jul. 1951, S. Hisamatsu leg. (EUMJ);

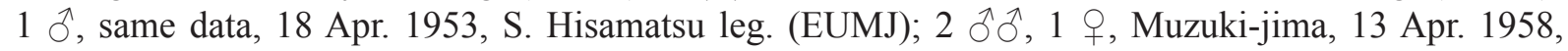
F. Takechi leg. (EUMJ). - Kochi Pref.: 4 $\widehat{\jmath}, 5$ 우오 Nankoku-shi, estuary of Monobe-gawa, 29 Jun.

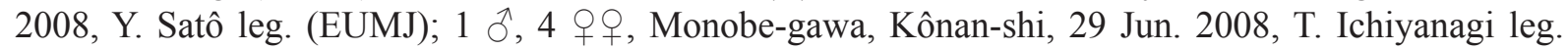
(EUMJ); 1 ㅇ, Yasuda-chô, Aki-shi, 29 Jun. 2008, T. Ichiyanagi leg. (EUMJ).

JAPAN, Kyushu: Saga Pref.: 1 ô, Kyogatake, 3-4 Aug. 1974, Y. Notsu leg. (EUMJ).

JAPAN, Ogasawara Isls.: 1 đ, 6 우 (already reported by Satô et al. 2006), Yome-jima, 15 Apr. 2000, T. Ohbayashi leg. (EUMJ).

RUSSIA: 1 §, 2 우우, Sakhalin, Adachi Isshiki leg. (SEHU). 


\section{Redescription}

Male

Body (Fig. 1E) oval, shining, with gray pubescence. Coloration of head, antennomeres IV-XI, pronotum and elytra black; antennomeres I-III and elytral fascia yellow; legs yellow, except for femora partly infuscate.

Head densely punctate; eyes oval, prominent; HL/HW 0.60-0.78 (0.67). Antennae(Fig. 4E) long, reaching base of elytra; antennomere III (Fig. 6E) broad bean-like shaped, excavate on dorsal surface; L/W $=1.05$; approximate ratio of each antennomere $(\mathrm{n}=1)$ as $5.2: 1.0: 6.3: 1.5: 1.7: 1.7: 1.7: 1.7: 1.7: 1.8: 2.7$. Pronotum rather densely punctate at lateral parts, rugosely so before hind angles, more or less sparsely so in middle part; PW/PL 1.07-1.42 (1.21). Elytra oval, broadened posteriorly, densely punctate; elytral fascia situated just before middle, widened at suture and lateral margins; EL/EW 1.21-1.52 (1.37); EL/ PL 2.45-3.26 (2.75); EW/PW 1.48-1.91 (1.66); TL/EW 1.99-2.49 (2.28). Aedeagus about $0.70 \mathrm{~mm}$ in AL. Gonoporal piece (Fig. 10E) straight, slender, $0.5 \mathrm{~mm}$ in GL; GL/AL 0.71. Semi-gonoporal piece and ligula absent. Spinous area (Fig. 9E) densely covered with long spines; spinous plate large and ovate.

\section{Female}

Similar to male in general appearance (Fig. 2E); coloration of antennae yellow, with apical 5 or 6 segments black; HL/HW 0.60-0.88 (0.69); PW/PL 1.04-1.38 (1.17); EL/EW 1.18-1.52 (1.33); EL/PL 2.38-3.00 (2.74); EW/PW 1.57-1.91 (1.75); TL/EW 1.99-2.46 (2.21). Antennae long; antennomere III short, distinctly expanded laterally, shallowly concave in dorsal portion, $\mathrm{L} / \mathrm{W}=1.91$; approximate ratio of each antennomere $(\mathrm{n}=1)$ as $5.5: 1.0: 3.5: 1.7: 1.7: 1.7: 1.7: 1.7: 1.7: 1.7: 2.7$. Hind wings absent.

\section{Measurements}

Male $(\mathrm{n}=20)$ : TL 2.73-4.17 (3.13) mm; HL 0.47-0.75 (0.56) mm; HW 0.76-0.99 (0.83) mm; PW 0.75-1.05 (0.85) mm; PL 0.55-0.87 (0.70) mm; EL 1.59-2.60 (1.92) mm; EW 1.12-1.79 (1.40) mm.

Female (n = 20): TL 2.82-4.22 (3.30) mm; HL 0.49-0.75 (0.57) mm; HW 0.73-1.00 (0.83) mm; PW 0.72-1.10 (0.86) mm; PL 0.64-0.88 (0.73) mm; EL 1.67-2.60 (2.00) mm; EW 1.29-2.00 (1.50) mm.

Distribution (Fig. 11B)

Japan (Hokkaido, Honshu, Shikoku, Kyushu, Shôdo-shima, Ogasawara Isls.: Yome-jima); Korea; Russia (Far East, Sakhalin [NR]).

\section{Remarks}

Female is apterous. The specimens from Hokkaido have a larger body, but the other features are quite the same as other specimens.

\section{Biological notes}

The larva of this species was described in detail by Asano (2013) and showed foetometamorphosis. This species inhabits beach plant communities (Asano 2013), and can be collected by sweeping beach plants (Fig. 12B).

Intybia takaraensis (Nakane, 1955)

[Japanese name: Ruri-ki-obi-jôkai-modoki]

Figs 1F, 2F, 4F, 5F, 6F, 8C, 9F, 10F, 11B

Laius takaraensis Nakane, 1955b: 376 [type locality: Takarajima Is., Tokara Isls.]. 
Laius takaraensis - Nakane 1957: 13, fig. 4. — Nakane 1963: 186, pl. 93, fig. 13. — Nakane 1983: 164. — Satô 1985: 164, pl. 26, fig. 7. — Satô 1989: 366.

Intybia takaraensis - Wittmer 1997: 181, abb. 39-46. — Mayor 2007: 417 [catalogued]. — Yoshitomi \& Hayashi 2011: 19. - Tshernyshev 2012: 575-587, table 1. — Plonski 2013: 66.

Laius tokaraensis Nakane 1956: 33 [misspelling].

\section{Diagnosis}

Coloration of body black with bluish luster on head, pronotum and elytra; elytra with a wide yellow fascia; antennomere III heart-like shape; gonoporal piece straight, slender, slightly curved basally; semigonoporal piece and ligula absent; spinous area (Fig. 9F) densely covered with long spines and with oval spinous plate.

\section{Material examined}

JAPAN, Honshu: Gifu Pref.: 1 O̊, Gifu, Japan, 25 Jun. 1947, D. Matusita leg. (EUMJ). - Aichi Pref.: 2 우, Yahagi-gawa, Murataka, Anjô-shi, 23 Sep. 1998 and 17 Jul. 1999, M. Hasegawa leg. (EUMJ). Mie Pref.: 1 q, Yokkaichi, 5 Jun. 1979, H. Nagai leg. (EUMJ); 1 §ૈ, same data, Aug. 1978, H. Nagai leg. (EUMJ); 1 ㅇ, Yokkaichi, Saburô-chô, 12 Jul. 1982, B. Tanaka leg. (EUMJ). - Shimane Pref.: 1 ô, 5 우, Tsunozu, Gotsu, 19 Aug. 2015, H. Yoshitomi leg. (EUMJ); 1 q, st. 24, 12 Jul. 2012, H. Yoshitomi leg. (EUMJ).

JAPAN, Shikoku: Ehime Pref.: 1 ð, Nakajima, 13 Oct. 1957, F. Takechi leg. (EUMJ); 11 $q$, Nishihabu-

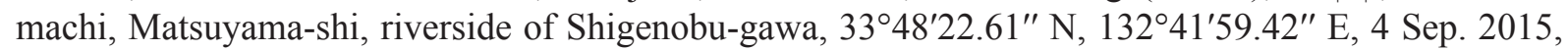

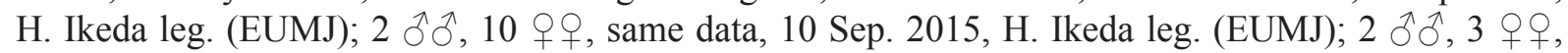
Shioya-kaigan, Kitagawara, Masaki-chô, Iyo-gun, estuary of Shigenobu-gawa, 20 Apr. 2015, H. Ikeda leg. (EUMJ).

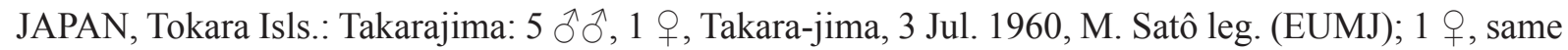
data, 3 Jun. 1962, M. Satô leg. (EUMJ); 1 ㅇ, same data, 4 Jun. 1962, M. Satô leg. (EUMJ); 3 đ̃ ô, 2 우우, same data, 5 Jun. 1962, M. Satô leg. (EUMJ); 1 ㅇ, same data, 6 Jun. 1962, M. Satô leg. (EUMJ); 1 đ̃, 4 q , s same data, 7 Jun. 1962, M. Satô leg. (EUMJ); 1 đ̃, 2 q $q$, same data, 8 Jun. 1962, M. Satô leg. (EUMJ).

JAPAN, Amami-Ôshima: Amami Isls.: Tokunoshima: 6 §ðึ, 10 우, Senma Beach, 20 Jun. 2001, M. Satô leg. (EUMJ). 1 đ, Koniya, 20 Apr. 1954, S. Takagi leg. (SEHU).

\section{Redescription}

Male

Body (Fig. 1F) oval, shining, sparsely covered with fulvous hairs. Coloration of head, pronotum and elytra black with bluish luster; antennae yellow, with apical 8 or 9 segments black; elytra with a wide yellow fascia just before the middle; tibiae, trochanters, apex of coxae, fore femora; apical portion of middle femora yellow.

Head moderately densely punctate; eyes oval and prominent laterally; HL/HW $0.62-0.82$ (0.69). Antennae (Fig. 4F) short, reaching base of elytra; antennomere I stout; antennomere III (Fig. 6F) heartlike shape, deeply concave in dorso-mesal portion; $\mathrm{L} / \mathrm{W}=1.12$; approximate ratio of each antennomere $(\mathrm{n}=1)$ as $9.7: 1.0: 12.7: 2.3: 2.3: 2.3: 3.0: 2.7: 2.7: 2.7: 4.3$. Pronotum transverse, broader than head; sides subparallel in the anterior half, then sinuately and strongly curved posteriorly; base slightly but broadly emarginated; surface rather densely punctate at the sides, rugosely so before hind angles, more or less sparsely and finely punctate in middle; PW/PL 1.13-1.40 (1.22). Elytra oval, moderately convex, broadened posteriorly, densely punctate at lateral parts; EL/EW 1.15-1.48 (1.36); EL/PL 2.39-3.07 
(2.68); EW/PW 1.48-1.83 (1.60); TL/EW 1.92-2.42 (2.28). Aedeagus about $0.73 \mathrm{~mm}$ in AL. Gonoporal piece (Figs 8C, 10F) straight, slender, slightly curved in basally, $0.44 \mathrm{~mm}$ in GL; GL/AL 0.60. Semigonoporal piece and ligula absent. Spinous area (Fig. 9F) densely covered with long spines; spinous plate large and ovate.

\section{Female}

Similar to male in general appearance (Fig. 2F); coloration of antennae yellow, with apical 7 or 8 segments black; HL/HW 0.54-0.68 (0.61); PW/PL 1.14-1.37 (1.24); EL/EW 1.22-1.53 (1.38); EL/ PL 2.60-3.03 (2.79); EW/PW 1.5-1.81 (1.63); TL/EW 1.99-2.41 (2.22). Antennae (Fig. 5F) long; antennomere III long, expanded laterally, shallowly concave in dorsal part; $\mathrm{L} / \mathrm{W}=2.00$; approximate ratio of each antennomere $(\mathrm{n}=1)$ as $9.7: 1.0: 6.7: 3.0: 3.0: 3.3: 3.3: 3.3: 3.3: 2.7: 5.0$.

\section{Measurements}

Male (n=18): TL 2.97-3.54 (3.18) mm; HL 0.50-0.62 (0.56) mm; HW 0.73-0.91 (0.81) mm; PW 0.77-1.03 (0.87) mm; PL 0.60-0.82 (0.72) mm; EL 1.67-2.14 (1.90) mm; EW 1.27-1.70 (1.40) mm.

Female (n=20): TL 2.78-3.26 (3.01) mm; HL 0.39-0.52 (0.47) mm; HW 0.71-0.85 (0.77) mm; PW 0.75-0.95 (0.83) mm; PL 0.62-0.75 (0.67) mm; EL 1.73-2.04 (1.87) mm; EW 1.17-1.58 (1.36) mm.

\section{Distribution (Fig. 11B)}

Japan (Honshu, Shikoku, Kyushu, Shôdo-shima, Takara-jima, Tokuno-shima [NR], Amami-Ôshima [NR]); China (Fujian, NE China), Russia (Primorye, Wrangel Is.).

\section{Remarks}

This species is closely similar to Intybia niponica (Lewis, 1895), and differs from it in the coloration of the body with a bluish luster and the shape of the male antennomere III.

\section{Biological notes}

This species inhabits beach plant communities or grasslands areas near large rivers.

$$
\begin{gathered}
\text { Intybia kishiii (Nakane, 1955) } \\
\text { [Japanese name: Beni-obi-jôkai-modoki] } \\
\text { Figs 1G, 2G, 4G, 5G, 6G, 8D, 9G, 10G, 11B }
\end{gathered}
$$

Laius kishiii Nakane, 1955a: 33 [type locality: Hozukyo, Kyoto].

Laius kishiii - Nakane 1957: 13. — Nakane 1963: 186, pl. 93, fig. 17. — Satô 1985: 165. — Nakane 1983: 164. - Satô 1989: 366.

Intybia kishiii - Wittmer 1997: 191. — Mayor 2007: 417 [catalogued]. — Yoshitomi \& Hayashi 2011: 19.

\section{Diagnosis}

Coloration of head, pronotum and elytra black with bluish luster; head narrow; eyes not prominent; elytral fascia reddish orange, widened at suture and lateral margins; gonoporal piece straight, slender, with straight basal projection; semi-gonoporal piece, ligula and spinous plate absent.

\section{Material examined}

JAPAN, Honshu: Gifu Pref.: 1 + , Suhara, 24 Jun. 1956, K. Ohbayashi leg. (EUMJ). - Okayama Pref.: 1 ô, Kurashiki-shi, Mizue, 17 Aug. 2007, H. Suenaga leg. (HSJ). - Hiroshima Pref.: 1 + , Miyoshi-shi, Miwaka, 13 Aug. 1980, T. Inakura leg. (EUMJ); 1 đ̂, Mihara-shi, Yahata-chô, Honjo, 28 Jun. 2013, H. Suenaga leg. (HSJ). 
JAPAN, Shikoku: Tokushima Pref.: 1 ㅇ, Tsuda Beach, 10 Jul. 1967, M. Sakai leg. (EUMJ). - Ehime Pref.: 1 $\widehat{\jmath}$, Ôzu, riverside of Hijikawa, Nakamura, 3 Jun. 2001, M. and A. Sakai leg. (EUMJ); 1 , same data, 7 Oct. 2001, Y. Kikuhara leg. (EUMJ); 1 J, Shigenobu-gawa, 27 Jun. 2010, R. Okano leg. (EUMJ). - Kochi Pref.: 1 Ĵ, 1 q, Nankoku-shi, estuary of Monobe-gawa, 29 Jun. 2008, Y. Satô leg.

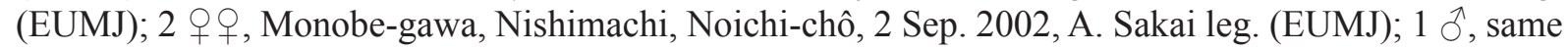
data, 4 Oct. 2002, C. Takahashi leg. (EUMJ); 1 O, 3 우오, same data, 6 Jul. 2002, C. Takahashi leg. (EUMJ); 1 , same data, 14 Aug. 2002, D. Miura leg. (EUMJ); 1 \%, same data, 4 Oct. 2002, A. Kitahara leg. (EUMJ); 1 ○, 1 क, Tosa-shi, estuary of Niyodo-gawa, 1 Jul. 2003, M. Sakai leg. (EUMJ); 1 ○, 2 우, same data, 5 Oct. 2002, T. Kurihara leg. (EUMJ); 2 우, Niyodo-gawa, Kada-Campsite, Ino-chô, 5 Oct. 2002, Y. Kikuhara leg. (EUMJ); 1 , same data, 13 Jul. 2002, Y. Kikuhara leg. (EUMJ); 1 ô, same data, 13 Jul. 2002, C. Takahashi leg. (EUMJ); 1 +, Haruno-chô, Niyodogawa-Ohashi, 13 Jul. 2002, C. Takahashi leg. (EUMJ).

\section{Redescription}

\section{Male}

Body (Fig. 1G) slightly elongate, gently convex, shining, bearing suberect pallid setae, sparsely covered with long erect setae. Coloration of head, antennomeres IV-XI, pronotum, elytra and legs black with bluish luster; antennomeres I-III reddish yellow; maxillary and labial palpi yellow; elytral fascia reddish orange.

Head narrowed anteriorly, with a deep longitudinal sulcus in middle, minutely punctate; eyes rather large, oval, not prominent; HL/HW 0.88-1.16 (1.00). Antennae (Fig. 4G) long, reaching base of elytra; antennomere III (Fig. 6G) subtrapezoidal, deeply concave in dorso-mesal portion; L/W $=0.81$; approximate ratio of each antennomere $(\mathrm{n}=1)$ as $7.0: 1.0: 6.5: 2.5: 2.3: 2.3: 2.5: 2.5: 2.5: 2.5: 3.7$. Pronotum convex, closely punctate in anterior parts, densely so in lateral parts; PW/PL 1.00-1.16 (1.07). Elytra oblong-ovate, slightly broadened posteriorly, rather coarsely and closely punctate in middle, finely and sparsely so at base and before apex; elytral fascia situated just before middle, widened at suture and lateral margins; EL/EW 1.47-1.71 (1.59); EL/PL 2.70-3.24 (2.94); EW/PW 1.53-1.89 (1.69); TL/EW 2.43-2.78 (2.53). Legs rather slender. Aedeagus about $0.85 \mathrm{~mm}$ in AL. Gonoporal piece (Figs 8D, 10G) straight, slender, with straight basal projection, $0.53 \mathrm{~mm}$ in GL; GL/AL 0.49. Semi-gonoporal piece, ligula and spinous plate absent. Spinous area (Fig. 9G) densely covered with short spines.

\section{Female}

Similar to male in general appearance (Fig. 2G); coloration of antennae yellow, with apical 7 segments black; HL/HW 0.77-1.07 (0.89); PW/PL 0.95-1.21(1.07); EL/EW 1.42-1.82 (1.55); EL/PL 2.69-3.48 (2.99); EW/PW 1.53-2.03 (1.81); TL/EW 2.26-2.81 (2.47). Antennae (Fig. 5G) long; antennomere III short, distinctly expanded laterally, shallowly concave in dorsal portion, $\mathrm{L} / \mathrm{W}=2.00$; approximate ratio of each antennomere $(\mathrm{n}=1)$ as $5.0: 1.0: 3.3: 2.5: 2.2: 2.0: 2.2: 2.0: 2.2: 1.8: 3.2$.

\section{Measurements}

Male (n = 8): TL 3.15-3.65 (3.38) mm; HL 0.49-0.71 (0.58) mm; HW 0.55-0.61 (0.57) mm; PW 0.70-0.86 (0.77) mm; PL 0.62-0.77 (0.71) mm; EL 1.92-2.22 (2.09) mm; EW 1.19-1.50 (1.32) mm.

Female ( $\mathrm{n}=19)$ : TL 3.08-4.20 (3.58) mm; HL 0.50-0.68 (0.57) mm; HW 0.56-0.70 (0.65) mm; PW 0.69-0.90 (0.80) mm; PL 0.63-0.83 (0.75) mm; EL 1.88-2.85 (2.25) mm; EW 1.22-1.70 (1.45) mm.

Distribution (Fig. 11B)

Japan (Honshu, Shikoku, Kyushu); Korea. 


\section{Remarks}

This species is distinct in having a narrow head and eyes that are not prominent.

\section{Biological notes}

This species inhabits grasslands at riversides.

Intybia kawasakii (Nakane, 1956)

[Japanese name: Okinawa-obi-jôkai-modoki]

Figs $1 \mathrm{H}, 2 \mathrm{H}, 4 \mathrm{H}, 5 \mathrm{H}, 11 \mathrm{~A}$

Laius kawasakii Nakane, 1956: 162 [type locality: Hedozaki, Kunigamison, Okinawa].

Laius kawasakii - Nakane 1957: 14, fig.5. — Nakane 1963: 186, pl. 93, fig. 18. — Satô 1985: 165. — Nakane 1983: 164. — Satô 1989: 366.

Intybia kawasakii - Wittmer 1997: 191. — Mayor 2007: 417 [catalogued]. — Yoshitomi \& Hayashi 2011: 19.

\section{Diagnosis}

Elytra with a pale yellow transverse fascia narrowly interrupted at suture, just before the middle; male antennomere III ear-like shaped.

\section{Type material examined}

Holotype

JAPAN: Ô, [Hedozaki, Kunigamison, Okinawa, 13 Jun. 1955, R. KAWASAKI.] (SEHU).

\section{Paratypes}

JAPAN: 3 우, same data as for the holotype (SEHU).

\section{Redescription}

\section{Male}

Body (Fig. 1H) oblong oval, shining, sparsely covered with gray pubescence. Coloration of head, pronotum and elytra black; antennae yellow, but apical 6 or 7 segments black; elytral fascia pale yellow, narrowly interrupted at suture; legs yellow; base of femora dark; hind femur and tibia black.

Head densely punctate; eyes normal, prominent; HL/HW 0.58. Antennae (Fig. 4H) long, reaching base of elytra; antennomere I long and stout; antennomere III ear-like shaped, deeply concave in dorso-mesal portion; $\mathrm{L} / \mathrm{W}=1.77$; approximate ratio of each antennomere $(\mathrm{n}=1)$ as $5.60: 1.00: 8.48: 1.16: 1.24: 1.24: 1.40: 1.60: 1.24: 1.60: 2.40$. Pronotum transverse, sparsely punctured in middle, densely so in lateral and posterior parts; PL/PW 1.17. Elytra oblong-ovate, a little broader than prothorax, then gently dilated to behind middle, sparsely punctate; EL/EW 1.51; EL/PL 2.70; EW/ PW 1.52; TL/EW 2.43. Male genitalia not examined.

\section{Female}

Similar to male in general appearance (Fig. 2H); coloration of antennae yellow, with apical 5 or 6 segments black; HL/HW 0.64-0.76 (0.70); PW/PL 1.13-1.18 (1.15); EL/EW 1.50-1.65 (1.57); EL/PL 2.71-2.82 (2.78); EW/PW 1.45-1.61 (1.54); TL/EW 2.48-2.66 (2.54). Antennae (Fig. 5H) long; antennomere III distinctly expanded laterally, shallowly concave in dorsal portion; $\mathrm{L} / \mathrm{W}=2.07$; approximate ratio of each antennomere $(\mathrm{n}=1)$ as $7.11: 1.00: 5.00: 2.24: 2.24: 2.11: 1.97: 2.18: 2.19: 2.37: 3.42$. 


\section{Measurements}

Male(n=1): TL3.04mm; HL 0.45 mm; HW0.77 mm; PW 0.82 mm; PL 0.7 mm;EL1.89 mm; EW $1.25 \mathrm{~mm}$.

Female $(\mathrm{n}=3)$ : TL 3.60-3.90 (3.79) mm; HL 0.58-0.63 (0.61) mm; HW 0.83-0.90 (0.88) mm; PW 0.90-1.00 (0.9) mm; PL 0.80-0.87 (0.84) mm; EL 2.17-2.45 (2.34) mm; EW 1.45-1.57 (1.49) mm.

\section{Distribution (Fig. 11A)}

Japan (Okinawa-jima).

\section{Remarks}

This species is rare and no additional specimens besides the type series have been collected. This species was recorded from Kyushu by Satô (1989) and Yoshitomi \& Hayashi (2011), but we regard this as a simple mistake.

\section{Biological notes}

The type series was collected from the northernmost cape of the island, and this species probably inhabits beach plant communities.

\section{Discussion}

The genus Intybia is divided into some species groups (Wittmer 1997; Plonski \& Geiser 2014). Wittmer (1997) regarded the seven Japanese species as being included in the pelegrini species group (= Gruppe 2). Based on the construction of the endophallic structure in the seven species (except for I. kawasakii, for which the endophallic structure could not be examined), the Japanese species are divided into the following two species groups:

The histrio group (histrio, tsushimensis, donan)

This group is proposed here, and is characterized by the following structures: male antennomere III with a projection near the base; elytra fascia does not widen at the suture; endophallic sclerites are composed of one gonoporal piece, one semi-gonoporal piece, and ligula; spinous plate absent.

The pelegrini group (pelegrini, niponica, takaraensis, kishiii)

This group was proposed by Wittmer (1997) for 26 Asian species and is redefined in this paper. It is characterized by the following structures: male antennomere III with or without a projection near the base; elytra fascia widened at the suture; endophallic sclerites composed of one gonoporal piece and 0-2 spinous plate(s); semi-gonoporal piece and ligula absent. Intybia kawasakii is expected to belong to this species group from the shape of the antennomere III.

The Japanese members of this group are subdivided into three subgroups as follows:

Subgroup 1 (pelegrini): with two spinous plates.

Subgroup 2 (niponica, takaraensis): with one spinous plate.

Subgroup 3 (kishiii): spinous plate absent.

\section{Acknowledgements}

We thank Dr Masahiro Ohara (SEHU) and Dr Munetoshi Maruyama (KUM) for their help in studying the museum specimens. We also thank Mr Yudai Ogata (Kinki University), Mr Naomichi Tsuji (Kyushu University), and Mr Haruki Suenaga (Tsuyama City) for examination of their private collections, and Mr Hiroaki Ohashi (Ehime University) for identification of Ardisia sieboldii. 


\section{References}

Asano M. 2013. Early instar larvae of Intybia niponicus (Lewis) (Coleoptera, Malachiidae) and comparison with a clerid 1st instar: The foetomorphic larva in Malachiidae, II. Japanese Journal of Systematic Entomology 19 (1): 21-27.

Asano M. 2015. Taxonomic notes on some malachiid beetles of Taiwan, with description of a new species of the genus Intybia Pascoe (Coleoptera: Malachiidae). Japanese Journal of Systematic Entomology 21 (1): $77-82$.

Asano M. \& Kawashima I. 2010. Discovery of the genus Dicranolaius (Coleoptera, Malachiidae) from Japan with description of a new species. Japanese Journal of Systematic Entomology 16 (2): 261-266.

Champion G.C. 1921. Notes on various african and asiatic species of Laius, Guérin, with an account at their accessory 0 -characters [Coleoptera]. The Annals and Magazine of Natural History 9 (7): 322-343. https://doi.org/10.1080/00222932108632526

Greiner J. 1937. Pars 159. Malachiidae. In: Schenkling S. (ed.) Coleopterorum Catalogus. Dr. W. Junk, 's Gravenhage.

Kiesenwetter E.A. H. von. 1874. Die Malacodermen Japans nach dem Ergebnisse der Sammulungen des Herrn G. Lewis währead der Jahre 1869-1871. Berliner Entomologische Zeitschrift 18: 241-288. https://doi.org/10.1002/mmnd.18740180302

Korea National Arboretum. 2014. Korean Plant Name Index: advice on online citation formats [online]. Available from http://www.nature.go.kr/ekbi/insct/clss/selectInsctStndaNmSrch.do [accessed 3 Aug. 2016].

Lewis G. 1893. A list of Coleoptera new to the fauna of Japan, with notices of unrecorded synonyms. The Entomologist 26: 150-153.

Lewis G. 1895. On the Daschillidae and malacoderm Coleoptera of Japan. The Annals and Magazine of Natural History (6) 16: 98-122, pl. 6. https://doi.org/10.1080/00222939508680233

Mayor A. J. 2007. Malachiidae. In: Löbl I. \& Smetana A. (ed.) Catalogue of Palaeartic Coleoptera, Vol.4, pp. 415-454. Apollo Books, Vester Skerninge. https://doi.org/10.1163/9789004260894

Miyatake M. \& Kobayashi T. 1950. Notes on the Coleoptera-fauna of Mt. Ishizuchi and its range (Shikoku, Japan). Bulletin of the Takarazuka Insectarium 73: 1-20. [In Japanese.]

Nakane T. 1955a. New or little-known Coleoptera from Japan and its abject regions XII. The Scientific Reports of the Saikyo University (Natural Science and Living Science) (A) 2 (1): 24-42.

Nakane T. 1955b. Marine insects of the Tokara Islands V. Three new species of the genus Laius Guerin from Kyusyu and the Tokara Islands, with notes on a species from Mariana. Publication of the Seto Mariana Biological Laboratory 4 (2-3): 373-378.

Nakane T. 1956. New or little-known Coleoptera from Japan and its abject regions, XIII. The scientific Reports of the Saikyo University (Natural Science and Living Science) (A) 2: 29-44, 159-174.

Nakane T. 1957. On Japanese Malachiinae (Coleoptera, Melyrdae) (2). Akitsu 6: 13-14.

Nakane T. 1963. Melyridae. In: Nakane T. Ohbayashi K. \& Kurosawa Y. (eds), Iconographia Insectorum Japonicorum Colore Naturali Edita 2: 186, pl. 93. Hokuryu-kan. [In Japanese.]

Nakane T. 1983. Notes on Japanese Melyridae (Coleoptera). Kitakyushu no Kontyu 30 (3): 161-166.

Pascoe F.P. 1866. Notices of new or little-known genera and species of Coleoptera. Journal of Entomology 2: 443-493. 
Pic M. 1910. Coléoptères exotiques nouveaux ou peu connus. L' Échange, Revue Linnéenne 26: 60-63.

Pic M. 1921. Nouveautés diverses. Mélanges exotico-entomologiques 33 : 1-32.

Plonski I.S. 2013. Studies on the genus Intybia Pascoe (Coleoptera: Malachiidae) I. Some nomenclatorial acts and faunistic records. Zeitschrift der Arbeitsgemeinschaft Österreichischer Entomologen 65: 61-68.

Plonski I.S. 2014a. Studies on the genus Intybia Pascoe, part II. Faunistic and taxonomic notes, with description of a new species of the I. plagiata-group (Coleoptera: Malachiidae). Koleopterologische Rundschau 84: 313-320.

Plonski I.S. 2014b. Studies on the genus Intybia Pascoe (Coleoptera: Malachiidae) IV. Notes on the fauna of the Philippines. Zeitschrift der Arbeitsgemeinschaft Österreichischer Entomologen 66: 39-45.

Plonski I.S. \& Geiser M. 2014. Studies on the genus Intybia Pascoe (Coleoptera: Malachiidae) III. On Intybia rubrithorax (Pic) and related taxa. Zeitschrift der Arbeitsgemeinschaft Österreichischer Entomologen 66: 31-38.

Satô M. 1985. Melyridae. In: Uéno S. Kurosawa Y. \& Satô M. (eds), The Coleoptera of Japan in Color, 3: 164-165, pl. 26. Hoikusha, Osaka. [In Japanese.]

Satô M. 1989. Melyridae. In: Hirahima Y. (et al.) A Check List of Japanese Insects 1: 366. [In Japanese.]

Satô M., Yoshitomi H. \& Ohbayashi T. 2006. Notes on Melyridae (Coleoptera) of Micronesia. Elytra, Tokyo 34: 343-351.

Tshernyshev S.E. 2009. A contribution to the fauna of soft winged flower beetles (Coleoptera, Malachiidae) of the Russian Far East. Eurosian Entomological Journal 8 (1): 35-37.

Tshernyshev S.E. 2012. A review of soft-winged flower beetle fauna (Coleoptera, Malachiidae) of North Asia. Eurosian Entomological Journal 11 (6): 575-587.

Wittmer W. 1995. Zur Kenntnis der Familie Malachiidae (Coleoptera), 2. Beitrag. Entomologica Basilensia 18: 287-391.

Wittmer W. 1997. Zur Kenntnis der Gattungen Intybia Pascoe und Stenolaius Wittmer (Coleoptera, Malachiidae). Japanese Journal of Systematic Entomology 3 (2): 181-211.

Wittmer W. 1999. Zur Kenntnis der Familie Malachiidae (Coleoptera), 3. Beitrag. Entomologica Basilensia 21: 171-252.

Yano T. 1957. [A check list of Shikoku Malacodermata -collection record of Coleoptera in Matsuyama neighborhood (5)-]. Ageha (4): 22-29. [In Japanese]

Yoshitomi H. 2014. Comparative morphology of the endophallic structures of the genus Laius (Coleoptera, Melyridae), with the description of three new species. European Journal of Taxanomy 97: 1-29. https://doi.org/10.5852/ejt.2014.97

Yoshitomi H. \& Hayashi N. 2011. List of the family Melyridae and its related families in Japan. Sayabane, New Series, Tokyo (2): 18-24. [In Japanese.]

Yoshitomi H. \& Lee C.-F. 2010. Revision of the Taiwanese and Japanese species of the genus Laius (Insecta: Coleoptera: Malachiidae). Zoological Studies 49 (4): 534-543. 
Manuscript received: 9 June 2016

Manuscript accepted: 24 August 2016

Published on: 20 June 2017

Topic editor: Gavin Broad

Desk editor: Jeroen Venderickx

Printed versions of all papers are also deposited in the libraries of the institutes that are members of the EJT consortium: Muséum national d'Histoire naturelle, Paris, France; Botanic Garden Meise, Belgium; Royal Museum for Central Africa, Tervuren, Belgium; Natural History Museum, London, United Kingdom; Royal Belgian Institute of Natural Sciences, Brussels, Belgium; Natural History Museum of Denmark, Copenhagen, Denmark; Naturalis Biodiversity Center, Leiden, the Netherlands; Museo Nacional de Ciencias Naturales-CSIC, Madrid, Spain; Real Jardín Botánico de Madrid CSIC, Spain. 